\title{
Functional relations in nineteen-vertex models with domain-wall boundaries
}

Cite as: J. Math. Phys. 60, 103509 (2019); https://doi.org/10.1063/1.5095588

Submitted: 12 March 2019 . Accepted: 19 September 2019 . Published Online: 31 October 2019

A. Bossart (D), and W. Galleas
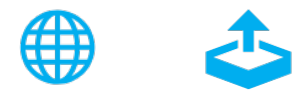

\section{ARTICLES YOU MAY BE INTERESTED IN}

A novel examination of the similarity between a perturbed hydrogen atom and an anharmonic oscillator

Journal of Mathematical Physics 60, 102102 (2019); https://doi.org/10.1063/1.5086981

Two-dimensional hydrogen-like atom in a constant magnetic field

Journal of Mathematical Physics 60, 102101 (2019); https://doi.org/10.1063/1.5100734

Contact Hamiltonian systems

Journal of Mathematical Physics 60, 102902 (2019); https://doi.org/10.1063/1.5096475

\section{Journal of}

Mathematical Physics

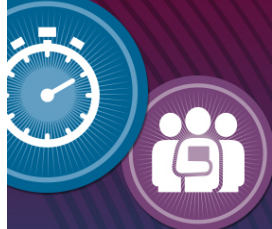

READY TO SUBMIT YOUR RESEARCH?

There are many reasons to publish with us. 


\title{
Functional relations in nineteen-vertex models with domain-wall boundaries
}

\author{
Cite as: J. Math. Phys. 60, 103509 (2019); doi: 10.1063/1.5095588 \\ Submitted: 12 March 2019 - Accepted: 19 September 2019 • \\ Published Online: 31 October 2019
}

\author{
A. Bossart ${ }^{1,2, a)}$ (i) and W. Galleas ${ }^{1, b)}$

\section{AFFILIATIONS} \\ ${ }^{1}$ Institut für Theoretische Physik, Eidgenössische Technische Hochschule Zürich, Wolfgang-Pauli-Strasse 27, \\ 8093 Zürich, Switzerland \\ ${ }^{2}$ Laboratory of Wave Engineering, École Polytechnique Fédérale de Lausanne, ELB O30 Station 11, \\ $\mathrm{CH}-1015$ Lausanne, Switzerland
}

a) Electronic mail: aleksi.bossart@epfl.ch
b) Electronic mail: galleasw@phys.ethz.ch

\section{ABSTRACT}

This work is concerned with functional properties shared by partition functions of nineteen-vertex models with domain-wall boundary conditions. In particular, we describe both Izergin-Korepin and Fateev-Zamolodchikov models with the aforementioned boundary conditions and show that their partition functions are governed by a system of functional equations originating from the associated Yang-Baxter algebra.

Published under license by AIP Publishing. https://doi.org/10.1063/1.5095588

\section{INTRODUCTION}

Vertex models of statistical mechanics can be regarded as a generalization of the ice model ${ }^{1,2}$ proposed in the early 1930 s aiming to describe the entropy of ice as its temperature goes to zero. In the case of ice, we are actually considering $\mathrm{H}_{2} \mathrm{O}$ molecules arranged in a crystalline structure, and it is natural to suppose other molecular systems can also be described in the same way. For instance, this is the case of the $\mathrm{KH}_{2} \mathrm{PO}_{4}$ molecule covered by the KDP model. ${ }^{3}$ References 4 and 5 discuss other variants of the ice model. The aforementioned molecular structures are all particular cases of the well-known six-vertex model, which turns out to be a two-dimensional abstraction of the former using concepts of graph theory. More accurately, the six-vertex model consists of a collection of colored graphs embedded in a twodimensional lattice where each vertex has a degree of four or one. As for the edges, each one can then assume two distinct configurations or colors. By allowing each edge to assume three distinct colors, we then have the so-called nineteen-vertex model as a possible two-dimensional lattice system generalizing the ideas of the six-vertex model.

\section{A. Integrable nineteen-vertex model}

As a matter of fact, the denomination nineteen-vertex model is very broad and one still needs to declare the statistical weights for each allowed graph configuration, in addition to the boundary conditions under consideration, in order to have the model fully defined. For instance, the previously mentioned six-vertex model is not a generic one as its statistical weights are carefully chosen in such a way that the model's partition function exhibits special properties; on several occasions, these special properties have allowed the exact computation

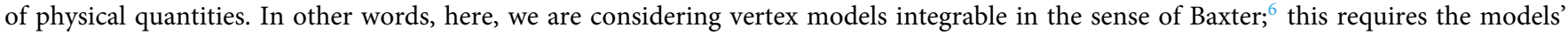
statistical weights to satisfy the Yang-Baxter equation.

As for the symmetric six-vertex model, there is essentially only one solution to the associated Yang-Baxter equation. However, a similar uniqueness statement does not hold for generic two-dimensional vertex models. For instance, to the best of our knowledge, the main representatives of nineteen-vertex models solving the Yang-Baxter equation correspond to 
- the Izergin-Korepin (IK) model,

- the Fateev-Zamolodchikov (FZ) model,

and solutions based on the $q$-deformed Lie superalgebras $U_{q}[\widehat{\mathfrak{o s p}}(1 \mid 2)], U_{q}\left[\widehat{\mathfrak{s l}}^{(2)}(1 \mid 2)\right]$, and $U_{q}\left[\widehat{\mathfrak{d o p p}}^{(2)}(2 \mid 2)\right]$. $^{9-12}$ In the present paper, we shall restrict our attention to the Izergin-Korepin (IK) and Fateev-Zamolodchikov (FZ) models, which also exhibit an underlying quantum affine Lie algebra $U_{q}[\widehat{\mathfrak{G}}] \cdot{ }^{13,14}$ In the case of the IK model, we have $\widehat{\mathfrak{G}}=A_{2}^{(2)}$, while $\widehat{\mathfrak{G}}=B_{1}^{(1)}$ for the FZ model.

\section{B. Boundary conditions}

After having the statistical weights of a vertex model fixed, one still needs to define appropriate boundary conditions in order for the model's partition function to be completely defined. Interestingly, different choices of boundary conditions not only influence the physical properties of the vertex model in the thermodynamical limit ${ }^{15}$ but also drastically change the kind of mathematical problem one needs to deal with in order to obtain the sought partition function in closed form.

For instance, by choosing periodic boundary conditions, one can resort to the Kramers and Wannier transfer matrix technique, ${ }^{16,17}$ and the evaluation of the model's partition function can be translated into the eigenvalue problem for the associated transfer matrix. As for the integrable nineteen-vertex models described above, the transfer matrix eigenvalue problem can be tackled through Tarasov's formulation of the Algebraic Bethe Ansatz (ABA). ${ }^{18}$ However, there still exist several other classes of boundary conditions which render vertex models of interest from both physics and mathematics perspectives. For instance, among the possible choices of boundaries, the so-called domain-wall boundary conditions are given special emphasis in this work. As for two-dimensional vertex models, domain-wall boundaries were introduced by Korepin in Ref. 19 as a tool for studying scalar products of Bethe vectors. However, it was already realized in Ref. 19 that these types of boundary conditions give rise to genuine vertex models which deserve independent attention. Hence, given the rich physical and mathematical structures associated with the six-vertex model with such types of boundary conditions, it is natural to wonder if we can extend the findings of the previous studies to more sophisticated two-dimensional vertex models. In this way, we find nineteen-vertex models to be natural targets as they, similarly to the six-vertex model, also constitute pillar supporting hierarchies of integrable systems of statistical mechanics.

\section{Previous results}

The literature devoted to nineteen-vertex models with domain-wall boundaries is to date quite modest when compared to the one studying the six-vertex model. In the case of the six-vertex model, some unusual physical behavior has been noticed and this was mainly due to Izergin's determinantal representation for the model's partition function. ${ }^{20}$ For instance, Izergin's formula has possibilitated the study of the influence of boundary conditions in the thermodynamical limit of the six-vertex model ${ }^{15,21-23}$ and the formation of limit shapes associated with spatial separation of phases. ${ }^{24,25}$ However, it is important to remark that several other determinantal representations are also available, ${ }^{26-28}$ as well as multiple contour integrals representations. ${ }^{29,30}$ In this way, it is compelling to try to extend the results available for the six-vertex model to nineteen-vertex models in order to further our understanding of the role played by boundary conditions in the thermodynamical limit of two-dimensional lattice models.

As for the FZ model with domain-wall boundaries, a determinantal representation has been obtained in Ref. 31 by identifying the FZ model with a spin-1 version of the six-vertex model. However, the problem is not that simple in the case of the IK model and a determinantal formula has been obtained in Ref. 32 only for a special value of the anisotropy parameter.

\section{Our approach}

The determinantal formulas of Refs. 31 and 32, obtained, respectively, for the FZ model and for a special case of the IK model, result from recurrence relations satisfied by the models' partition functions. This recursive approach is essentially the same method originally put forward by Korepin in Ref. 19 for the six-vertex model, ultimately leading to Izergin's representation. ${ }^{20}$ However, one inherent step of this approach is making an educated guess for the sought partition function, which can then be shown to correspond to the actual partition function if it satisfies the aforementioned recurrence relations in addition to extra properties. In this way, the construction of such determinantal representations can elude us in more sophisticated models.

An alternative method based on functional equations was put forward in Ref. 33 and subsequently refined in a series of works. ${ }^{34,29,30,35,27}$ We shall refer to this approach as the Algebraic-Functional (AF) method, and it is responsible, among other results, for the construction of single determinant representations for the elliptic solid-on-solid model with domain-wall boundaries, ${ }^{35,27}$ which were previously thought to not admit such types of representations. Hence, given the above-described scenario, the extension of the AF method to nineteen-vertex models with domain-wall boundaries is a sound problem which has been discussed in the present paper.

\section{E. Outline}

We have organized this paper as follows. In Sec. II, we describe the algebraic formulation of integrable nineteen-vertex models, with special emphasis on the IK and FZ models as they are the specific vertex models we will be considering in the present work. In Sec. II, we shall also precise the boundary conditions relevant to our forthcoming analysis and present properties expected from the models' partition functions. Section III is then devoted to the formulation of the AF method to both IK and FZ models with domain-wall boundaries in a 
unified way. Functional equations governing our models' partition functions are then derived and inspected in Sec. IV. Section V is then left for concluding remarks, and technical details and extra results are presented in Appendixes A and B.

\section{NINETEEN-VERTEX MODELS}

This work is concerned with nineteen-vertex models with particular domain-wall boundary conditions; however, our analysis will require a more general formulation allowing also for other choices of boundaries. In this way, we shall start this section with the introduction of conventions and concepts which will assist us throughout Secs. III-V.

Write $[n]:=\{0,1, \ldots, n, n+1\}$, and let $\operatorname{Rect}_{K, L}:=[K] \times[L] \subseteq \mathbb{Z}_{\geq 0}^{2}$ denote a two-dimensional lattice with a bulk grid formed by the crossing of $K$ rows and $L$ columns. In addititon, let

$$
\mathcal{G}=\bigcup_{\substack{i \in[K] \backslash\{0, K+1\} \\ j \in[L] \backslash\{0, L+1\}}} g_{i, j}
$$

be a graph built from the juxtaposition of local subgraphs $g_{i, j}$. The latter consists of $g_{i, j}=\left(\mathcal{V}_{i, j}, \mathcal{E}_{i, j}\right)$ with vertices $\mathcal{V}_{i, j}=\left\{v_{i, j}, v_{i, j-1}\right.$, $\left.v_{i, j+1}, v_{i-1, j}, v_{i+1, j}\right\}$ and edges

$$
\mathcal{E}_{i, j}=\left\{d_{v_{i, j}}\left(v_{i, j-1}\right), d_{v_{i, j}}\left(v_{i, j+1}\right), d_{v_{i, j}}\left(v_{i-1, j}\right), d_{v_{i, j}}\left(v_{i+1, j}\right)\right\}
$$

In (2.2), we have used $d_{v_{i, j}}\left(v_{k, l}\right)=d_{v_{k, l}}\left(v_{i, j}\right)$ to denote the edge connecting generic vertices $v_{i, j}$ and $v_{k, l}$. We then embed $\mathcal{G}$ on Rect ${ }_{K, L}$ by identifying $v_{i, j}$ with $(i, j) \in \operatorname{Rect}_{K, L}$.

Next, we would like to promote $\mathcal{G}$ to an edge-colored graph $\mathcal{G}^{*}$ obtained through the assignment $d_{v_{i, j}}\left(v_{k, l}\right) \mapsto d_{v_{i, j}}^{(\alpha)}\left(v_{k, l}\right)$ for all edges in $\mathcal{G}$. The label $\alpha$ is then introduced to characterize the color or configuration assigned to a given edge. Here, we are interested in the socalled nineteen-vertex models, and in that case, each edge $d_{v_{i j}}^{(\alpha)}\left(v_{k, l}\right)$ in $\mathcal{G}^{*}$ can take three distinct configurations. For instance, we shall write

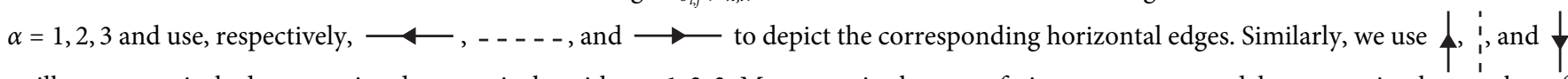
to illustrate vertical edges associated, respectively, with $\alpha=1,2,3$. Moreover, in the case of nineteen-vertex models, we restrict the number of possible edge-colored graphs $g_{i, j}$ to nineteen among the $3^{4}=81$ possibilities. The allowed graphs $g_{i, j}$ are then depicted in Fig. 1 .

Remark 2.1 (Conservation of arrows). The diagrammatic representations shown in Fig. 1 make manifest an important conservation law in nineteen-vertex models. For instance, one can readily see in Fig. 1 that all graphs $g_{i, j}$ have the same number of arrows pointing inward and outward. Here, we refer to this rule as conservation of arrows.

At the end of the day, one would like to associate a partition function with the graph $\mathcal{G}^{*}$ embedded on Rect $K, L$. This will additionally require the introduction of boundary conditions and statistical weights for local graph configurations $g_{i, j}$. We shall return to this issue in Subsections II A-II G.

\section{A. Algebraic formulation}

Write $\mathcal{R}\left(\lambda_{i}, \mu_{j}\right)_{\alpha_{i, j}, \beta_{i+1, j}}^{\alpha_{i, j}, \beta_{i, j}}$ for the statistical weight associated with the local edge-colored graph $g_{i, j}$, as shown in Fig. 2. In addition, let us introduce vectors

$$
\begin{aligned}
& \vec{\alpha}_{j}:=\left(\alpha_{1, j}, \alpha_{2, j}, \ldots, \alpha_{K, j}\right), \\
& \vec{\beta}_{i}:=\left(\beta_{i, 1}, \beta_{i, 2}, \ldots, \beta_{i, L}\right) .
\end{aligned}
$$

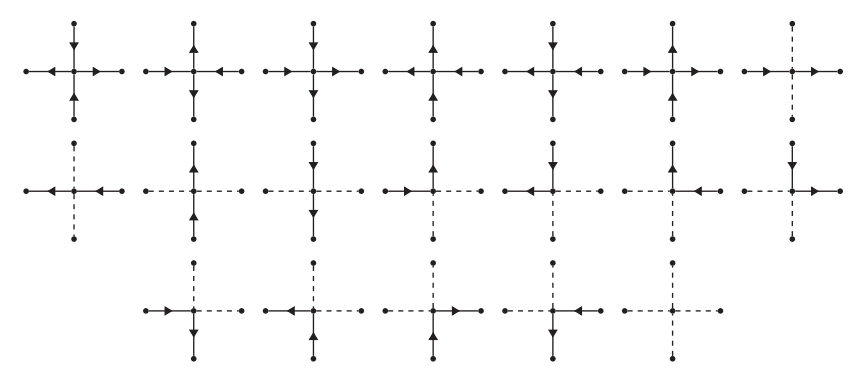

FIG. 1. Graphs $g_{i, j}$ in nineteen-vertex models. 


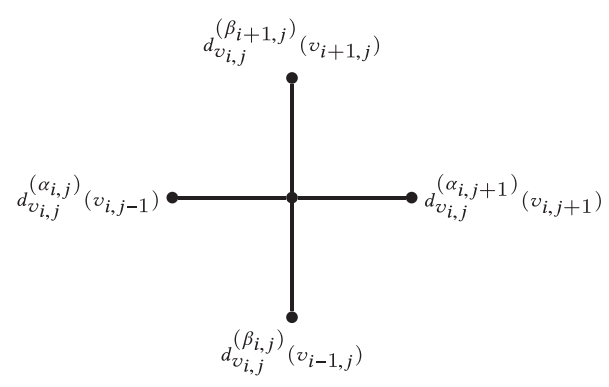

FIG. 2. Local edge-colored graph $g_{i, j}$.

In this way, we can define a partition function with fixed boundary conditions for $\mathcal{G}^{*}$ on $\operatorname{Rect}_{K, L}$ as

$$
Z_{\vec{\beta}_{0}}^{\vec{\beta}_{K+1}}\left(\vec{\alpha}_{0} \mid \vec{\alpha}_{L+1}\right):=\sum_{\alpha_{i, j}, \beta_{i, j} \in\{1,2,3\}} \prod_{\substack{i \in[K] \backslash\{0, K+1\} \\ j \in[L] \backslash\{0, L+1\}}} \mathcal{R}\left(\lambda_{i}, \mu_{j}\right)_{\alpha_{i, j}, \beta_{i+1, j}}^{\alpha_{i, j+1}, \beta_{i, j}} .
$$

The RHS of (2.4) looks overwhelming at first sight, but, fortunately, it can be rewritten in an operatorial manner along the lines of the Kramers and Wannier transfer matrix technique. In order to present such operatorial formulation, let us introduce vector spaces $\mathbb{V}=\mathbb{V}_{a}=\mathbb{V}_{i} \simeq \mathbb{C}^{3}$ for $i=1,2, \ldots, L$ and let $\left\{e_{1}, e_{2}, e_{3}\right\}$ be standard basis vectors of $\mathbb{C}^{3}$. More precisely, we take

$$
e_{1}:=\left(\begin{array}{l}
1 \\
0 \\
0
\end{array}\right), \quad e_{2}:=\left(\begin{array}{l}
0 \\
1 \\
0
\end{array}\right), \quad \text { and } \quad e_{3}:=\left(\begin{array}{l}
0 \\
0 \\
1
\end{array}\right)
$$

In addition, we write $E_{\alpha, \alpha^{\prime}} \in \operatorname{End}\left(\mathbb{C}^{3}\right)$ for unit matrices defined by $E_{\alpha, \alpha^{\prime}}\left(e_{\beta}\right):=\delta_{\alpha^{\prime}, \beta} e_{\alpha}$ for $\beta=1,2,3$. Next, we define the matrix $\mathcal{R}: \mathbb{C} \times \mathbb{C}$ $\rightarrow \operatorname{End}(\mathbb{V} \otimes \mathbb{V})$ as

$$
\mathcal{R}\left(\lambda_{i}, \mu_{j}\right):=\sum_{\substack{\alpha, \alpha^{\prime} \in\{1,2,3\} \\ \beta, \beta^{\prime} \in\{1,2,3\}}} \mathcal{R}\left(\lambda_{i}, \mu_{j}\right)_{\alpha, \beta}^{\alpha^{\prime}, \beta^{\prime}} E_{\alpha, \alpha^{\prime}} \otimes E_{\beta, \beta^{\prime}}
$$

Remark 2.2. The conservation of arrows pointed out in Remark 2.1 reflects in the $\mathcal{R}$-matrix formalism (2.6) by only allowing nonvanishing statistical weights $\mathcal{R}_{\alpha, \beta}^{\alpha^{\prime}, \beta^{\prime}}$ such that $\alpha+\beta=\alpha^{\prime}+\beta^{\prime}$.

Here, we intend to express the partition function (2.4) in terms of the $\mathcal{R}$-matrix (2.6). With that goal in mind, we then introduce the so-called monodromy matrix $\mathcal{T}: \mathbb{C} \times \mathbb{C}^{L} \rightarrow \operatorname{End}\left(\mathbb{V}_{a} \otimes \mathbb{V}_{\mathcal{Q}}\right)$ with $\mathbb{V}_{\mathcal{Q}}:=\otimes_{i=1}^{L} \mathbb{V}_{i}$. More precisely, we write

$$
\mathcal{T}\left(\lambda_{i} \mid\left\{\mu_{j}\right\}\right):=\prod_{1 \leq j \leq L} \mathcal{R}_{a j}\left(\lambda_{i}, \mu_{j}\right) \quad \in \operatorname{End}\left(\mathbb{V}_{a} \otimes \mathbb{V}_{1} \otimes \cdots \otimes \mathbb{V}_{L}\right)
$$

using the tensor leg notation. The monodromy matrix $\mathcal{T}$ can also be regarded as the matrix in $\operatorname{End}\left(\mathbb{V}_{a}\right)$ with entries in End $\left(\mathbb{V}_{\mathcal{Q}}\right)$. In this way, we also have

$$
\mathcal{T}\left(\lambda \mid\left\{\mu_{j}\right\}\right)=:\left(\begin{array}{lll}
\mathcal{A}_{1}(\lambda) & \mathcal{B}_{1}(\lambda) & \mathcal{B}_{2}(\lambda) \\
\mathcal{C}_{1}(\lambda) & \mathcal{A}_{2}(\lambda) & \mathcal{B}_{3}(\lambda) \\
\mathcal{C}_{2}(\lambda) & \mathcal{C}_{3}(\lambda) & \mathcal{A}_{3}(\lambda)
\end{array}\right)
$$

deliberately omitting the dependence on parameters $\mu_{j} \in \mathbb{C}$ in the RHS. Consequently, we shall then use the notation $\mathcal{T}_{\alpha}^{\beta}$ to refer to the entry of (2.8) corresponding to the element $E_{\alpha, \beta} \in \operatorname{End}\left(\mathbb{V}_{a}\right)$. Next, we define vectors

$$
\left|\vec{\beta}_{i}\right\rangle:=\bigotimes_{j=1}^{L} e_{\beta_{i, j}} \quad \in \mathbb{V}_{\mathcal{Q}}
$$

completing, in this way, the ingredients required to reformulate (2.4). Then, using (2.6)-(2.8), we can rewrite our partition function with fixed boundary conditions in terms of entries of the monodromy matrix $\mathcal{T}$ as 


$$
Z_{\vec{\beta}_{0}}^{\vec{\beta}_{K+1}}\left(\vec{\alpha}_{0} \mid \vec{\alpha}_{L+1}\right)=\left\langle\vec{\beta}_{K+1}\left|\prod_{1 \leq i \leq K}^{\leftarrow} \mathcal{T}\left(\lambda_{i} \mid\left\{\mu_{j}\right\}\right)_{\alpha_{i, 0}}^{\alpha_{i L+1}}\right| \vec{\beta}_{0}\right\rangle
$$

The statistical weights associated with configurations of graphs $g_{i, j}$ are encoded in the $\mathcal{R}$-matrix (2.6). Although they are still generic up to this point, integrability in the sense of Baxter requires the $\mathcal{R}$-matrix (2.6) to satisfy the Yang-Baxter equation. The following discussion will then be restricted to integrable nineteen-vertex models, and in that case, we can consider $\mathcal{R}(\lambda, \mu)=\mathcal{R}(\lambda-\mu)$ and use the convention

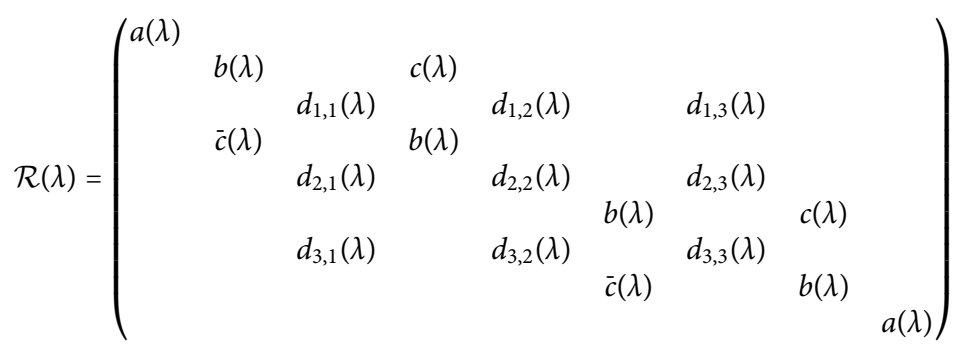

in order to ease our presentation. In Subsection II B, we shall then discuss two distinct sets of statistical weights satisfying the Yang-Baxter equation.

\section{B. The IK and FZ models}

Strictly speaking, integrability in statistical mechanics is not a well defined concept as it is in classical mechanics. ${ }^{36}$ Nevertheless, Baxter's concept of commuting transfer matrices ${ }^{6}$ has played a major role in identifying two-dimensional lattice models whose physical properties can be computed exactly. Here, we will be considering nineteen-vertex models integrable in the sense of Baxter, and this requires the $\mathcal{R}$-matrix (2.11) to satisfy the Yang-Baxter equation. More precisely, we will focus on statistical weights $a, b, c, \bar{c}$, and $d_{i, j}$ constrained by

$$
\mathcal{R}_{12}\left(\lambda_{1}-\lambda_{2}\right) \mathcal{R}_{13}\left(\lambda_{1}-\lambda_{3}\right) \mathcal{R}_{23}\left(\lambda_{2}-\lambda_{3}\right)=\mathcal{R}_{23}\left(\lambda_{2}-\lambda_{3}\right) \mathcal{R}_{13}\left(\lambda_{1}-\lambda_{3}\right) \mathcal{R}_{12}\left(\lambda_{1}-\lambda_{2}\right)
$$

in $\operatorname{End}\left(\mathbb{V}_{1} \otimes \mathbb{V}_{2} \otimes \mathbb{V}_{3}\right)$. In contrast to the six-vertex model, there are several solutions of (2.12) corresponding to nineteen-vertex models. In what follows, we shall describe two of them, namely, the Izergin-Korepin (IK) and the Fateev-Zamolodchikov (FZ) models.

The IK model originally appeared as the quantization of integrable structures associated with the Shabat-Mikhailov model. ${ }^{7}$ The latter is a relativistic field theory in $1+1$ dimensions whose integrability, in the classical sense, is ensured by the existence of a Lax pair and a classical $r$-matrix. The $\mathcal{R}$-matrix of the IK model then arises as the quantization of the aforementioned classical $r$-matrix. On the other hand, the FZ model first appeared within the context of factorized scattering. ${ }^{8}$ More precisely, the $\mathcal{R}$-matrix of the FZ model was originally obtained as the $\mathcal{S}$-matrix of a quantum field theory enjoying $\mathrm{C}, \mathrm{P}, \mathrm{T}$, and $\mathrm{U}(1)$ symmetries. Those symmetries are able to fix the $\mathcal{S}$-matrix up to a large extent, while the remaining part is then fixed by the Yang-Baxter equation.

The quantum group structure underlying the $\mathcal{R}$-matrices of the IK and FZ models was only later on unveiled in Ref. 14 . They correspond to the quantum affine Lie algebras $U_{q}\left[\widehat{A}_{2}^{(2)}\right]$ and $U_{q}\left[\widehat{B}_{1}^{(1)}\right]$, respectively, and this algebraic structure also allows the associated statistical weights to be presented in a unified manner. In this way, we have

$$
\begin{array}{ll}
a(\lambda)=\left(e^{2 \lambda}-\zeta\right)\left(e^{2 \lambda}-q^{2}\right), & b(\lambda)=q\left(e^{2 \lambda}-1\right)\left(e^{2 \lambda}-\zeta\right), \\
c(\lambda)=\left(1-q^{2}\right)\left(e^{2 \lambda}-\zeta\right), & \bar{c}(\lambda)=e^{2 \lambda}\left(1-q^{2}\right)\left(e^{2 \lambda}-\zeta\right),
\end{array}
$$

and

$$
d_{\alpha, \beta}(\lambda)=\left\{\begin{array}{lr}
q\left(e^{2 \lambda}-1\right)\left(e^{2 \lambda}-\zeta\right)+e^{2 \lambda}\left(q^{2}-1\right)(\zeta-1), & \alpha=\beta=\beta^{\prime}, \\
\left(e^{2 \lambda}-1\right)\left[\left(e^{2 \lambda}-\zeta\right)+e^{2 \lambda}\left(q^{2}-1\right)\right], & \alpha=\beta \neq \beta^{\prime}, \\
\left(q^{2}-1\right)\left[\zeta\left(e^{2 \lambda}-1\right) q^{(\alpha-\beta) / 2}-\delta_{\alpha \beta^{\prime}}\left(e^{2 \lambda}-\zeta\right)\right], & \alpha<\beta, \\
e^{2 \lambda}\left(q^{2}-1\right)\left[\left(e^{2 \lambda}-1\right) q^{(\alpha-\beta) / 2}-\delta_{\alpha \beta^{\prime}}\left(e^{2 \lambda}-\zeta\right)\right], & \alpha>\beta,
\end{array}\right.
$$


with $\alpha^{\prime}:=4-\alpha$. The parameter $\zeta$ is, in its turn, given by

$$
\zeta=\left\{\begin{array}{cc}
q, & \text { for FZ model } \\
-q^{3}, & \text { for IK model }
\end{array}\right.
$$

\section{Domain-wall boundaries}

In this subsection, we intend to specialize the partition function (2.10) to cases of interest in this work. For instance, our main goal here is to study the partition function (2.10) with $K=L$ and the particular boundary conditions characterized by

$$
\vec{\alpha}_{0}=\vec{\beta}_{0}=(1,1, \ldots, 1) \text { and } \quad \vec{\alpha}_{L+1}=\vec{\beta}_{L+1}=(3,3, \ldots, 3)
$$

We shall then simply write $\mathcal{Z}\left(\lambda_{1}, \lambda_{2}, \ldots, \lambda_{L}\right)$ for (2.10) with boundary conditions (2.16). The variables $\lambda_{j} \in \mathbb{C}$ are usually referred to as spectral parameters, but it is important to remark that $\mathcal{Z}$ also depends on $L$ variables $\mu_{j} \in \mathbb{C}$ commonly referred to as inhomogeneity parameters. Moreover, according to (2.13)-(2.15), the partition function $\mathcal{Z}$ also depends on the quantum deformation parameter $q=: e^{\gamma} \in \mathbb{C}$ and $\gamma$ will then be referred to as the anisotropy parameter. In order to describe the boundary conditions (2.16) in a more intuitive way, we have also presented a possible edge-colored graph $\mathcal{G}^{*}$ admitted by $\mathcal{Z}$ in Fig. 3.

Although this work is mainly concerned with the partition function $\mathcal{Z}$, our analysis will reveal that this partition function is intimately related to another two partition functions also obtained as specializations of $(2.10)$ with $K=L+1$. In this way, we write $\mathcal{F}\left(u_{1}, u_{2}, \ldots, u_{L-1} \mid\right.$ $\left.v_{1}, v_{2}\right)$ for (2.10) with $K=L+1, \lambda_{1}=v_{1}, \lambda_{2}=v_{2}, \lambda_{i+2}=u_{i}(i=1,2, \ldots, L-1)$, and boundary vectors

$$
\begin{aligned}
\vec{\alpha}_{0} & =(1,1, \ldots, 1,1), \\
\vec{\alpha}_{L+1} & =(2,2,3,3, \ldots, 3),
\end{aligned}
$$

$$
\begin{aligned}
\vec{\beta}_{0} & =(1,1, \ldots, 1), \\
\vec{\beta}_{K+1}, & =(3,3, \ldots, 3) .
\end{aligned}
$$

Similarly, we also define $\overline{\mathcal{F}}\left(v_{1}, v_{2} \mid u_{1}, u_{2}, \ldots, u_{L-1}\right)$ as the specialization of $(2.10)$ with $K=L+1, \lambda_{i}=u_{i}(i=1,2, \ldots, L-1), \lambda_{L}=v_{1}$, and $\lambda_{L+1}=v_{2}$. As for the boundary vectors, $\vec{\alpha}_{0}, \vec{\beta}_{0}$, and $\vec{\beta}_{K+1}$ are identical to the ones in (2.17), while $\vec{\alpha}_{L+1}=(3,3, \ldots, 3,2,2)$. A sample of graphs $\mathcal{G}^{*}$ giving rise to $\mathcal{F}$ and $\overline{\mathcal{F}}$ is then depicted in Fig. 4 .

For the sake of clarity, it is also useful to have $\mathcal{Z}, \mathcal{F}$, and $\overline{\mathcal{F}}$ expressed directly in terms of entries of the monodromy matrix (2.8). As for that, we introduce the simplified conventions

$$
\mathcal{A}_{1}(\lambda)=: \mathcal{A}(\lambda), \quad \mathcal{B}_{1}(\lambda)=: \mathcal{B}(\lambda), \quad \text { and } \quad \mathcal{B}_{2}(\lambda)=: \mathcal{E}(\lambda)
$$

as well as vectors $|0\rangle:=e_{1}^{\otimes L}$ and $|\overline{0}\rangle:=e_{3}^{\otimes L}$ in $\operatorname{End}\left(\mathbb{V}_{\mathcal{Q}}\right)$. The aforementioned partition functions are then given by the following expected values:

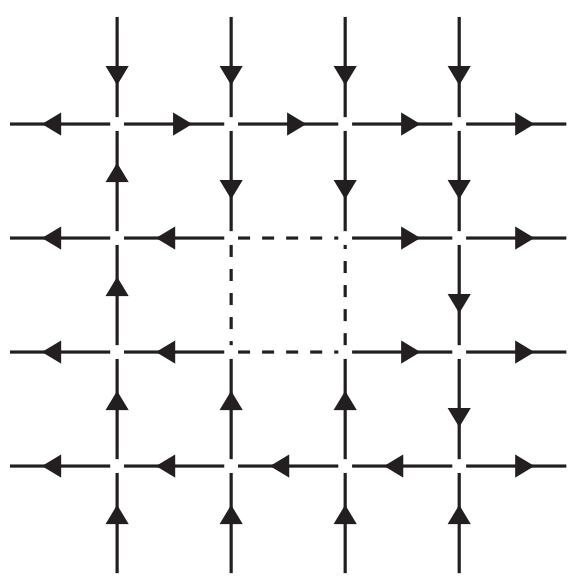

FIG. 3. Example of $\mathcal{G}^{*}$ in $\mathcal{Z}$ for $L=4$. 

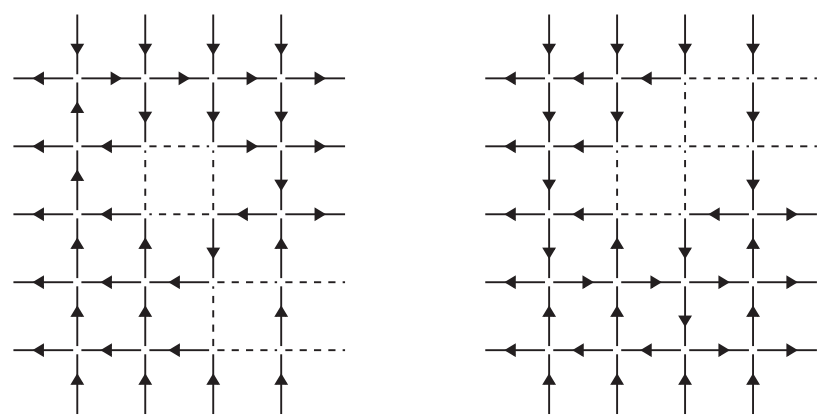

FIG. 4. Graphs $\mathcal{G}^{*}$ for $L=4$ associated with $\mathcal{F}$ (left) and $\overline{\mathcal{F}}$ (right).

$$
\begin{aligned}
\mathcal{Z}\left(\lambda_{1}, \lambda_{2}, \ldots, \lambda_{L}\right) & =\left\langle\overline{0}\left|\mathcal{E}\left(\lambda_{L}\right) \mathcal{E}\left(\lambda_{L-1}\right) \ldots \mathcal{E}\left(\lambda_{1}\right)\right| 0\right\rangle \\
\mathcal{F}\left(u_{1}, u_{2}, \ldots, u_{L-1} \mid v_{1}, v_{2}\right) & =\left\langle\overline{0}\left|\mathcal{E}\left(u_{L-1}\right) \mathcal{E}\left(u_{L-2}\right) \ldots \mathcal{E}\left(u_{1}\right) \mathcal{B}\left(v_{2}\right) \mathcal{B}\left(v_{1}\right)\right| 0\right\rangle \\
\overline{\mathcal{F}}\left(v_{1}, v_{2} \mid u_{1}, u_{2}, \ldots, u_{L-1}\right) & =\left\langle\overline{0}\left|\mathcal{B}\left(v_{2}\right) \mathcal{B}\left(v_{1}\right) \mathcal{E}\left(u_{L-1}\right) \mathcal{E}\left(u_{L-2}\right) \ldots \mathcal{E}\left(u_{1}\right)\right| 0\right\rangle
\end{aligned}
$$

It is also important to remark here that having $\mathcal{Z}, \mathcal{F}$, and $\overline{\mathcal{F}}$ expressed as (2.19) will play a major role in our forthcoming analysis.

\section{Symmetries}

In Appendix $\mathrm{A}$, we have collected commutation relations satisfied by the operators $\mathcal{A}, \mathcal{B}$, and $\mathcal{E}$ built from the $\mathcal{R}$-matrix (2.11) for the IK and FZ models. Among such commutation relations, we have

$$
\mathcal{E}\left(\lambda_{i}\right) \mathcal{E}\left(\lambda_{j}\right)=\mathcal{E}\left(\lambda_{j}\right) \mathcal{E}\left(\lambda_{i}\right)
$$

which has immediate consequences for $\mathcal{Z}, \mathcal{F}$, and $\overline{\mathcal{F}}$. In order to examine such consequences, let us write $\mathfrak{S}_{n}$ for the symmetric group of degree $n$ on $\left\{\lambda_{1}, \lambda_{2}, \ldots, \lambda_{n}\right\}$. In addition to that, let $\pi_{i, j} \in \mathfrak{S}_{n}$ be a 2-cycle acting as permutation of variables $\lambda_{i}$ and $\lambda_{j}$. Therefore, due to the commutation relation (2.20), we immediately obtain $\pi_{i, j}(\mathcal{Z})=\mathcal{Z}$, which allows us to infer $\mathcal{Z}\left(\lambda_{1}, \lambda_{2}, \ldots, \lambda_{L}\right) \in \mathbb{C}\left[\lambda_{1}^{ \pm 1}, \lambda_{2}^{ \pm 1}, \ldots, \lambda_{L}^{ \pm 1}\right]^{\mathfrak{S}_{L}}$. In other words, the partition function $\mathcal{Z}$ is a symmetric function on all arguments $\lambda_{j}$.

On the other hand, according to formulas (2.19), the partition functions $\mathcal{F}$ and $\overline{\mathcal{F}}$ also involve the operator $\mathcal{B}$ whose commutation relations with $\mathcal{E}$ are sufficiently more involving. Hence, $\mathcal{F}$ and $\overline{\mathcal{F}}$ are not symmetric with respect to all of their arguments. However, they are clearly partially symmetric and we can also infer

$$
\mathcal{F}\left(\lambda_{1}, \ldots, \lambda_{L-1} \mid v_{1}, v_{2}\right), \overline{\mathcal{F}}\left(v_{1}, v_{2} \mid \lambda_{1}, \ldots, \lambda_{L-1}\right) \in \mathbb{C}\left[\lambda_{1}^{ \pm 1}, \lambda_{2}^{ \pm 1}, \ldots, \lambda_{L-1}^{ \pm 1}\right]^{\mathfrak{S}_{L-1}}\left[v_{1}^{ \pm 1}, v_{2}^{ \pm 1}\right]
$$

\section{E. Polynomial structure}

In Subsection II D, we have analyzed the behavior of the functions $\mathcal{Z}, \mathcal{F}$, and $\overline{\mathcal{F}}$ with respect to the action of the symmetric group $\mathfrak{S}_{n}$. In this way, we were able to infer the kind of function space the functions of interest belongs to. Here, we intend to further that analysis by examining in more detail the dependence of $\mathcal{Z}, \mathcal{F}$, and $\overline{\mathcal{F}}$ on the spectral parameters. For that, it is convenient to introduce variables $x:=e^{2 \lambda}$, $x_{i}:=e^{2 \lambda_{i}}, y_{1}:=e^{2 v_{1}}$, and $y_{2}:=e^{2 v_{2}}$.

Now, turning our attention to the statistical weights (2.13)-(2.15) associated with the IK and FZ models, we can readily see they are polynomials in $x$ of degree two, except for $d_{1,2}, d_{1,3}, d_{2,3}$, and $c$. In their turn, the latter are polynomials in $x$ of degree one. Therefore, we can conclude that the functions $\mathcal{Z}, \mathcal{F}$, and $\overline{\mathcal{F}}$ are polynomials in the appropriate variables, and in what follows, we intend to determine their polynomial degree.

In order to proceed, it is then useful to identify the graphs $g_{i, j}$ with their respective statistical weight (2.13)-(2.15). For that we write $w\left(g_{i, j}\right) \in\left\{a, b, c, \bar{c}, d_{i, j}\right\}$ and make this identification explicit in Figs. 5-8.

Lemma 2.3. The partition function $\mathcal{Z}\left(\lambda_{1}, \lambda_{2}, \ldots, \lambda_{L}\right)$ is a symmetric polynomial of degree $2 L-1$ in each variable $x_{i}=e^{2 \lambda_{i}}$ separately.

Proof. The polynomial structure is a direct consequence of (2.11), (2.8), (2.19), and (2.13)-(2.15), while the symmetry property with respect to the permutation of arguments has already been proved in Sec. II D. In addition, according to (2.19), one can see that the whole dependence of $\mathcal{Z}$ on a given variable $\lambda_{i}$ is contained in a single operator $\mathcal{E}\left(\lambda_{i}\right)$. Next, since $\mathcal{Z}$ is symmetric, it suffices to inspect its dependence on the variable $x_{1}$. The latter then arises from the statistical weights associated with the concatenation of graphs $g_{1, j}$ for $1 \leq j \leq L$, respecting the conservation of arrows discussed in Remarks 2.1 and 2.2. In this way, one only needs to inspect the contribution originated from the 


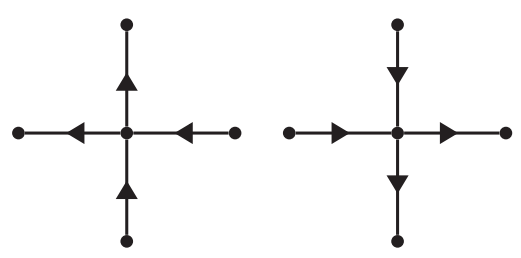

FIG. 5. Graphs $g_{i, j}$ with $w\left(g_{i, j}\right)=a$.

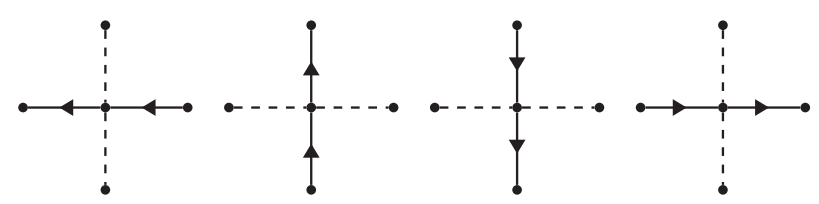

FIG. 6. Graphs $g_{i, j}$ with $w\left(g_{i, j}\right)=b$.

sequence

$$
w\left(g_{1,1}\right) \rightarrow w\left(g_{1,2}\right) \rightarrow \cdots \rightarrow w\left(g_{1, L}\right) .
$$

Due to the domain-wall boundary conditions, we have $w\left(g_{1,1}\right) \in\left\{d_{1,3}, c, a\right\}$, while $w\left(g_{1, L}\right) \in\left\{d_{1,3}, d_{2,3}, d_{3,3}\right\}$. On the same basis, we find the restrictions $w\left(g_{1, j}\right) \in\left\{d_{1,3}, d_{2,3}, c, a, b, d_{3,3}\right\}$ for $2 \leq j \leq L-1$. Next, we introduce the short-hand notation $\{\Lambda\}^{n}$ for the repeated sequence of $n$ terms $\{\Lambda\} \rightarrow\{\Lambda\} \rightarrow \cdots \rightarrow\{\Lambda\}$ of any element $\Lambda$. The latter will be useful when describing the possible sequences (2.22) arising under domain-wall boundary conditions. Then, given the above-described constraints, we have the following possible sequences:

(i) $\left\{d_{1,3}\right\} \rightarrow\left\{d_{3,3}\right\}^{L-1}$,

(ii) $\{c\} \rightarrow\{b\}^{n} \rightarrow\left\{d_{2,3}\right\} \rightarrow\left\{d_{3,3}\right\}^{L-n-2}, \quad 0 \leq n \leq L-2$,

(iii) $\{a\}^{1+n} \rightarrow\left\{d_{1,3}\right\} \rightarrow\left\{d_{3,3}\right\}^{L-n-2}$,

$$
0 \leq n \leq L-2,
$$

(iv) $\{a\}^{1+n} \rightarrow\{c\} \rightarrow\{b\}^{m} \rightarrow\left\{d_{2,3}\right\} \rightarrow\left\{d_{3,3}\right\}^{L-m-n-3}, \quad 0 \leq m \leq L-n-3,0 \leq n \leq L-2$.

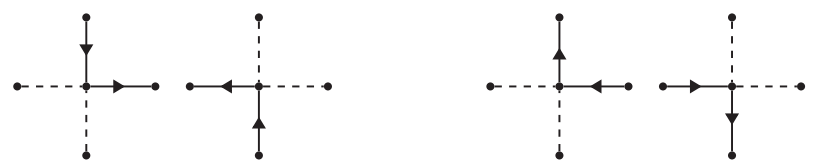

FIG. 7. Graphs $g_{i, j}$ with $w\left(g_{i, j}\right)=c$ (most left) and $w\left(g_{i, j}\right)=\bar{c}$ (most right).

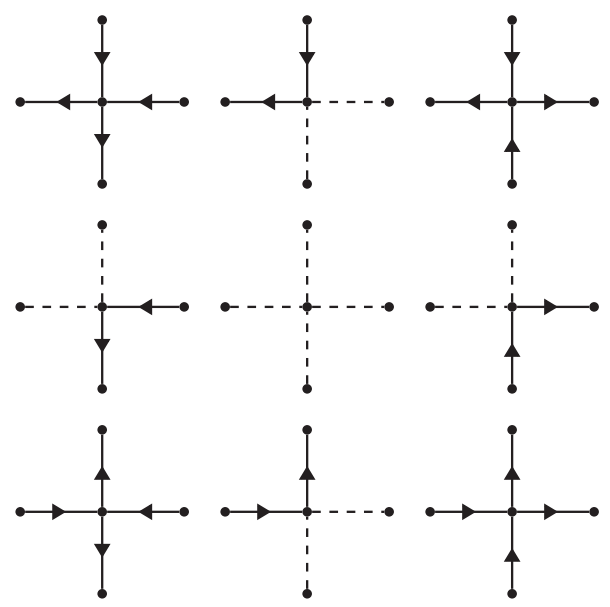

FIG. 8. Graphs $g_{k, l}$ with $w\left(g_{k, l}\right)=d_{i, j}$ at the ith row and jth column. 
The Sequences (i) and (iii) then give rise to polynomials in $x_{1}$ of degree $2 L-1$, while (ii) and (iv) contribute to polynomials of degree $2 L-2$. Therefore, we can conclude that $\mathcal{Z}$ is a polynomial in $x_{1}$ of degree $2 L-1$.

Remark 2.4. Alternatively, one could have similarly inspected the sequence $w\left(g_{L, 1}\right) \rightarrow w\left(g_{L, 2}\right) \rightarrow \cdots \rightarrow w\left(g_{L, L}\right)$ for the proof of Lemma 2.3.

Lemma 2.5. The functions $\mathcal{F}\left(\lambda_{1}, \lambda_{2}, \ldots, \lambda_{L-1} \mid v_{1}, v_{2}\right)$ and $\overline{\mathcal{F}}\left(v_{1}, v_{2} \mid \lambda_{1}, \lambda_{2}, \ldots, \lambda_{L-1}\right)$ are polynomials of degree $2 L-1$ in each variable $x_{i}=e^{2 \lambda_{i}}$ separately and also of degree $2 L-1$ in each variable $y_{i}=e^{2 v_{i}}$.

Proof. The dependence of $\mathcal{F}$ and $\overline{\mathcal{F}}$ on the variable $x_{i}$ follows straightforwardly from the analysis performed in the proof of Lemma 2.3 . Therefore, here we only need to examine the dependence on the variables $y_{1}$ and $y_{2}$.

We shall then start with the analysis of $\mathcal{F}$, and from (2.19), we can see that the whole dependence on $y_{1}$ is enclosed in the operator $\mathcal{B}\left(v_{1}\right)$. In this way, we only need to examine the contribution originating from the concatenation of graphs $g_{1, j}(j=1,2, \ldots, L)$ in order to determine the polynomial degree in the variable $y_{1}$. More precisely, here we also need to inspect the sequence (2.22) but now with possible statistical weights

$$
\begin{array}{lr}
w\left(g_{1,1}\right) \in\left\{d_{1,3}, c, a\right\}, & w\left(g_{1, L}\right), \in\{b, c\}, \\
w\left(g_{1, j}\right), \in\left\{d_{1,3}, d_{2,3}, d_{3,3}, a, b, c\right\}, & 2 \leq j \leq L-1,
\end{array}
$$

in order to comply with the required boundary conditions. In this way, we find the allowed sequences

(i) $\{c\} \rightarrow\{b\}^{L-1}$,

(ii) $\{a\}^{1+n} \rightarrow\{c\} \rightarrow\{b\}^{L-n-2} \quad 0 \leq n \leq L-2$.

Both sequences (i) and (ii) produce polynomials of degree $2 L-1$ in the variable $y_{1}$. Therefore, the dependence on $y_{1}$ stated in Lemma 2.5 is proved.

Next, we move on to the dependence on $y_{2}$. The latter is similarly obtained from the inspection of sequences

$$
w\left(g_{2,1}\right) \rightarrow w\left(g_{2,2}\right) \rightarrow \cdots \rightarrow w\left(g_{2, L}\right) .
$$

The allowed sequences (2.24) will depend strongly on possibilities (i) and (ii) for (2.22) due to conservation of arrows. In this way, we shall split our analysis and first consider scenario (i) for (2.22). In that case, we find the possible statistical weights

$$
\begin{array}{lrl}
w\left(g_{2,1}\right) \in\left\{b, d_{1,2}\right\}, & w\left(g_{2, L}\right) \in\{b, c\}, \\
w\left(g_{2, j}\right) \in\left\{d_{1,3}, d_{2,3}, d_{3,3}, a, b, c\right\}, & 2 \leq j \leq L-1,
\end{array}
$$

due to arrows conservation. The latter then yields the following possible sequences (2.24):

(i.a) $\quad\left\{d_{1,2}\right\} \rightarrow\{b\}^{L-1}$,

\begin{tabular}{|c|c|c|}
\hline $\begin{array}{l}\text { (ii.a) } \\
\text { (ii.b) } \\
\text { (ii.c) }\end{array}$ & $\begin{array}{l}\left\{d_{1,3}\right\} \rightarrow\left\{d_{3,3}\right\}^{r} \rightarrow\left\{d_{3,2}\right\} \rightarrow\{b\}^{L-r-2} \\
\{c\} \rightarrow\{b\}^{r} \rightarrow\left\{d_{2,2}\right\} \rightarrow\{b\}^{L-r-2} \\
\{c\} \rightarrow\{b\}^{r} \rightarrow\left\{d_{2,3}\right\} \rightarrow\left\{d_{3,3}\right\}^{s} \rightarrow\left\{d_{3,2}\right\} \rightarrow\{b\}^{L-r-s-3}, \\
\{a\}^{1+r} \rightarrow\left\{d_{1,3}\right\} \rightarrow\left\{d_{3,3}\right\}^{s} \rightarrow\left\{d_{3,2}\right\} \rightarrow\{b\}^{L-r-s-3}, \\
\{a\}^{1+r} \rightarrow\{c\} \rightarrow\{b\}^{s} \rightarrow\left\{d_{2,2}\right\} \rightarrow\{b\}^{L-r-s-3}, \\
\{a\}^{1+r} \rightarrow\{c\} \rightarrow\{b\}^{s} \rightarrow\left\{d_{2,3}\right\} \rightarrow\left\{d_{3,3}\right\}^{t} \rightarrow\left\{d_{3,2}\right\} \rightarrow\{b\}^{L-}\end{array}$ & $\begin{aligned} & 0 \leq r \leq L-2, \\
& 0 \leq r \leq L-2, \\
& 0 \leq s \leq L-r-3,0 \leq r \leq L-2, \\
& 0 \leq s \leq L-r-3,0 \leq r \leq L-2, \\
& 0 \leq s \leq L-r-3,0 \leq r \leq L-2, \\
& L-r-s-t-4, \quad 0 \leq t \leq L-r-s-4,\end{aligned}$ \\
\hline & & $0 \leq s \leq L-r-3,0 \leq r \leq L-2$ \\
\hline g & $\begin{array}{l}\{a\}^{1+r} \rightarrow\left\{d_{1,2}\right\} \rightarrow\{b\}^{L-r-2}, \\
\{a\}^{1+r} \rightarrow\{b\} \rightarrow\{c\} \rightarrow\{b\}^{L-r-3} \\
\{a\}^{1+r} \rightarrow\{b\} \rightarrow\{a\}^{s} \rightarrow\{c\} \rightarrow\{b\}^{L}\end{array}$ & $\leq s \leq L-r-3,0$ \\
\hline
\end{tabular}

(i.b) $\quad\{b\} \rightarrow\{a\}^{r} \rightarrow\{c\} \rightarrow\{b\}^{L-r-2} \quad 0 \leq r \leq L-2$.

The inspection of (2.24) under scenario (ii) is more involving but still doable. In that case, the statistical weights entering (2.24) are restricted to

$$
\begin{aligned}
w\left(g_{2,1}\right) & \in\left\{d_{1,3}, c, a\right\}, \\
w\left(g_{2, j}\right) & \in\left\{d_{1,3}, d_{2,3}, d_{3,3}, a, b, c\right\}, \\
w\left(g_{2, n+2}\right) & \in\left\{b, c, \bar{c}, d_{1,2}, d_{2,2}, d_{3,2}\right\} .
\end{aligned}
$$

Then, considering (2.26), we have the following allowed sequences (2.24): 
Except from (ii.c) and (ii.f), all the contributions arising from (i.a) to (ii.i) are polynomials in $y_{2}$ of degree $2 L-1$. As for (ii.c) and (ii.f), they give rise to polynomials of degree $2 L-2$. In this way, $\mathcal{F}\left(\lambda_{1}, \lambda_{2}, \ldots, \lambda_{L-1} \mid v_{1}, v_{2}\right)$ is a polynomial of degree $2 L-1$ in $y_{2}$. This concludes the proof of Lemma 2.5 for the function $\mathcal{F}$.

As for the function $\overline{\mathcal{F}}$, one needs to inspect the sequences

$$
w\left(g_{L+1,1}\right) \rightarrow w\left(g_{L+1,2}\right) \rightarrow \cdots \rightarrow w\left(g_{L+1, L}\right)
$$

and

$$
w\left(g_{L, 1}\right) \rightarrow w\left(g_{L, 2}\right) \rightarrow \cdots \rightarrow w\left(g_{L, L}\right)
$$

instead of (2.22) and (2.24), in order to analyze its dependence on $y_{1}$ and $y_{2}$. According to our boundary conditions, the possible sequences (2.27) and (2.28) can be obtained directly from (2.22) and (2.24) relevant to $\mathcal{F}$ by mapping each graph $g_{i, j}$ in the sequence to its counterpart with vertical edges flipped around the central vertex. We then arrive at the same conclusions for $\mathcal{F}$.

\section{F. Simple zeroes of $\mathcal{F}$ and $\overline{\mathcal{F}}$}

From expressions (2.19), one can promptly see that the sets of variables $\left\{u_{j}\right\}$ and $\left\{v_{j}\right\}$ entering the arguments of $\mathcal{F}$ and $\overline{\mathcal{F}}$ are not on equal footing. In particular, as previously discussed in Sec. II D, the functions $\mathcal{F}$ and $\overline{\mathcal{F}}$ are symmetric under the permutation of variables $u_{j}$, while a similar statement regarding the variables $v_{j}$ cannot be made. Here, however, we intend to show that the strategy used in the proof of Lemma 2.5 can still yield us more information on the structure of the aforementioned functions.

Lemma 2.6. The partition function $\mathcal{F}\left(\lambda_{1}, \ldots, \lambda_{L-1} \mid v_{1}, v_{2}\right)$ vanishes for the specializations $y_{1}=e^{2 \mu_{j}} \zeta(1 \leq j \leq L)$.

Proof. The dependence of $\mathcal{F}\left(\lambda_{1}, \ldots, \lambda_{L-1} \mid v_{1}, v_{2}\right)$ on $y_{1}=e^{2 v_{1}}$ is characterized by the allowed sequences (i) and (ii) described in the proof of Lemma 2.5. Those sequences contain only the statistical weights $b, c$, and $a$, which share the overall common factor $(x-\zeta)$. Therefore, the partition function $\mathcal{F}\left(\lambda_{1}, \ldots, \lambda_{L} \mid v_{1}, v_{2}\right)$ vanishes for the specialization $y_{1}=e^{2 \mu_{j}} \zeta$.

Corollary 2.7. The function $\mathcal{F}$ can be written as

$$
\mathcal{F}\left(\lambda_{1}, \lambda_{2}, \ldots, \lambda_{L-1} \mid v_{1}, v_{2}\right)=: \omega\left(y_{1}\right) \mathcal{H}\left(\lambda_{1}, \lambda_{2}, \ldots, \lambda_{L-1} \mid v_{1}, v_{2}\right)
$$

with

$$
\omega(y):=\prod_{j=1}^{L}\left(y-e^{2 \mu_{j}} \zeta\right)
$$

and $\mathcal{H}\left(\lambda_{1}, \lambda_{2}, \ldots, \lambda_{L-1} \mid v_{1}, v_{2}\right)$ being a polynomial of degree $L-1$ in $y_{1}$. The dependence on the other variables is still the same as of $\mathcal{F}$

Proof. Direct consequence of the polynomial structure described in Lemma 2.5 and the simple zeroes of Lemma 2.6.

Next, we turn our attention to the function $\overline{\mathcal{F}}\left(v_{1}, v_{2} \mid \lambda_{1}, \lambda_{2}, \ldots, \lambda_{L-1}\right)$ and our goal is to obtain analogous versions of Lemma 2.6 and Corollary 2.7. This can be obtained from the inspection of possible sequences (2.27) which, in their turn, can be directly read off from (i) and (ii) by flipping the vertical edges of the graphs $g_{i, j}$ entering those sequences around the central vertex. In this way, we obtain the following properties for the partition function $\overline{\mathcal{F}}$.

Lemma 2.8. The partition function $\overline{\mathcal{F}}\left(v_{1}, v_{2} \mid \lambda_{1}, \lambda_{2}, \ldots, \lambda_{L-1}\right)$ vanishes when $y_{2}=e^{2 \mu_{j}}$ for $1 \leq j \leq L$.

Proof. The dependence of $\overline{\mathcal{F}}$ on $y_{2}$ is characterized by the allowed sequences (2.27), which can be obtained from (i) and (ii) through the aforementioned flipping procedure. The latter is then mimicked by the maps $b \mapsto b, c \mapsto d_{2,3}$, and $a \mapsto d_{3,3}$, and we are left with the following sequences:

(iii) $\left\{d_{2,3}\right\} \rightarrow\{b\}^{L-1}$,

(iv) $\left\{d_{3,3}\right\}^{1+n} \rightarrow\left\{d_{2,3}\right\} \rightarrow\{b\}^{L-n-2}, \quad 0 \leq n \leq L-2$.

Similarly to the analysis performed for $\mathcal{F}$, one can now see (iii) and (iv) contain only the statistical weights $d_{2,3}, d_{3,3}$, and $b$. These weights, in their turn, share the overall common factor $(x-1)$ which implies that $\overline{\mathcal{F}}\left(v_{1}, v_{2} \mid \lambda_{1}, \lambda_{2}, \ldots, \lambda_{L-1}\right)$ vanishes when $y_{2}=e^{2 \mu_{j}}$. 
Corollary 2.9. The function $\overline{\mathcal{F}}$ can be written as

$$
\overline{\mathcal{F}}\left(v_{1}, v_{2} \mid \lambda_{1}, \lambda_{2}, \ldots, \lambda_{L-1}\right)=: \bar{\omega}\left(y_{2}\right) \overline{\mathcal{H}}\left(v_{1}, v_{2} \mid \lambda_{1}, \lambda_{2}, \ldots, \lambda_{L-1}\right)
$$

with

$$
\bar{\omega}(y):=\prod_{j=1}^{L}\left(y-e^{2 \mu_{j}}\right)
$$

and $\overline{\mathcal{H}}\left(v_{1}, v_{2} \mid \lambda_{1}, \lambda_{2}, \ldots, \lambda_{L-1}\right)$ being a polynomial of degree $L-1$ in the variable $y_{2}$.

Proof. Similarly to Corollary (2.7), formulas (2.31) and (2.32) are direct consequences of Lemmas 2.5 and 2.8.

\section{G. Initial condition}

Up to this point, we have collected definitions and properties associated with the partition functions $\mathcal{Z}, \mathcal{F}$, and $\overline{\mathcal{F}}$, and in Sec. III, we intend to put forward a functional approach for studying the aforementioned quantities. Our main goal here is to study the partition function $\mathcal{Z}$, but our framework will show such a function is closely related to $\mathcal{F}$ and $\overline{\mathcal{F}}$. In particular, using the AF method, we will find a linear functional equation characterizing $\mathcal{Z}$ and, as such, it will require an initial condition in order for the sought quantities to be fixed completely. In what follows, we shall then demonstrate the existence of a special point where $\mathcal{Z}$ can be easily evaluated.

Lemma 2.10 (Initial condition). As for the specializations $\lambda_{i}=\mu_{i}$, for $1 \leq i \leq L$, we have

$$
\mathcal{Z}\left(\mu_{1}, \mu_{2}, \ldots, \mu_{L}\right)=\prod_{i, j=1}^{L} a\left(\mu_{i}-\mu_{j}\right) .
$$

Proof. We first notice that the $\mathcal{R}$-matrix characterized by (2.6), (2.11), and (2.13)-(2.15) is regular in the sense that $\mathcal{R}_{i j}\left(\mu_{j}, \mu_{j}\right)=\mathcal{R}_{i j}(0)$ $=a(0) \mathcal{P}_{i j}$ with $\mathcal{P}_{i j}: \mathbb{V}_{i} \otimes \mathbb{V}_{j} \rightarrow \mathbb{V}_{j} \otimes \mathbb{V}_{i}$ being the permutation operator. In particular, as for $\mathcal{R}_{i j}\left(\mu_{j}, \mu_{j}\right)$, we only have contributions from the nine graphs $g_{i, j}$ depicted in Figs. 5 and 7 and the antidiagonal elements of Fig. 8. Hence, given the domain-wall boundary conditions, we have the single allowed sequence

$$
\begin{aligned}
\left\{a\left(\mu_{L-i+1}-\mu_{1}\right)\right\} & \rightarrow\left\{a\left(\mu_{L-i+1}-\mu_{2}\right)\right\} \rightarrow \cdots \rightarrow\left\{a\left(\mu_{L-i+1}-\mu_{L-i}\right)\right\} \\
& \rightarrow\left\{d_{1,3}\left(\mu_{L-i+1}-\mu_{L-i+1}\right)\right\} \rightarrow\left\{a\left(\mu_{L-i+1}-\mu_{L-i+2}\right)\right\} \\
& \rightarrow\left\{a\left(\mu_{L-i+1}-\mu_{L-i+3}\right)\right\} \rightarrow \cdots \rightarrow\left\{a\left(\mu_{L-i+1}-\mu_{L}\right)\right\}
\end{aligned}
$$

for the $i$ th row of our lattice under the specialization $\lambda_{i}=\mu_{L-i+1}$. For the sake of clarity, such a configuration is diagrammatically represented in Fig. 9 for $L=4$. Then, considering $d_{1,3}(0)=a(0)$ and that $\mathcal{Z}$ is a symmetric function, we immediately obtain formula (2.33).

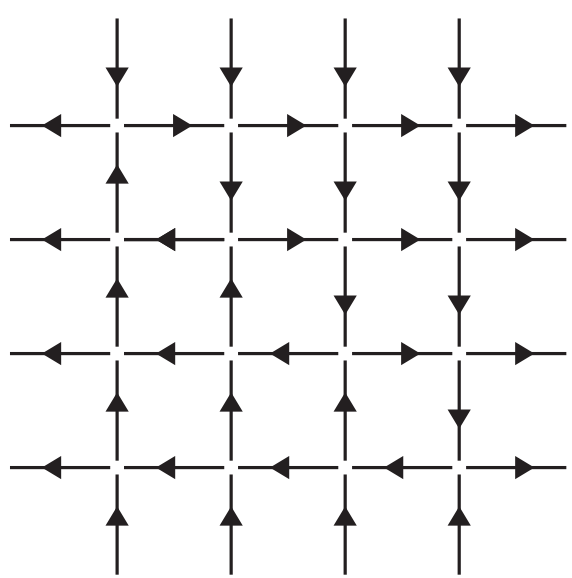

FIG. 9. Graph $\mathcal{G}^{*}$ in $\mathcal{Z}$ for $L=4$ and $\lambda_{i}=\mu_{i}$. 


\section{ALGEBRAIC-FUNCTIONAL APPROACH}

Section II is devoted to the description of nineteen-vertex models with domain-wall boundary conditions. In particular, we have discussed properties of the partition functions $\mathcal{Z}, \mathcal{F}$, and $\overline{\mathcal{F}}$ defined by (2.4) with boundary conditions (2.16) and (2.17). In addition to that, we have also rewritten the aforementioned partition functions in an operatorial manner in (2.19). The evaluation of such partition functions in closed form is certainly an important step toward the exact computation of physical properties of those systems and finding constraints fully characterizing $\mathcal{Z}, \mathcal{F}$, and $\overline{\mathcal{F}}$ is our present goal.

In Subsections II D-II G, we have derived a series of properties satisfied by our partition functions, but they are not enough to characterize the aforementioned quantities since, as polynomials, their complete determination requires fixing all their zeroes or coefficients. The latter is only partially achieved by the results of Sec. II D-II G, and in order to present enough constraints fixing our partition functions, here we intend to formulate the above problem in terms of functional equations along the lines of the Algebraic-Functional (AF) method. This framework has offered an alternative to Izergin and Korepin's method ${ }^{19,20}$ in the case of six-vertex models and generalizations, and it is based on the characterization of quantities of interest by means of functional equations originated from the Yang-Baxter algebra. Roughly speaking, the AF method is a framework aiming to convert algebraic relations into functional equations describing quantities of interest and, as for integrable vertex models, the so-called Yang-Baxter algebra is a suitable algebraic structure for that end.

\section{A. Yang-Baxter algebra}

Let us write $\mathcal{L}_{i} \in \operatorname{End}\left(\mathbb{V}_{i}\right)$ for a matrix with noncommutative entries fulfilling the relation

$$
\mathcal{R}_{i j}(x-y) \mathcal{L}_{i}(x) \mathcal{L}_{j}(y)=\mathcal{L}_{j}(y) \mathcal{L}_{i}(x) \mathcal{R}_{i j}(x-y) \quad \in \operatorname{End}\left(\mathbb{V}_{i} \otimes \mathbb{V}_{j}\right)
$$

with $\mathcal{R}_{i j}$ being previously defined in Sec. II. We then refer to (3.1) as the Yang-Baxter algebra and use $\mathscr{A}(\mathcal{R})$ to denote it. The partition functions discussed in Sec. II will then be related to a particular representation of $\mathscr{A}(\mathcal{R})$ consisting of a pair $\left(\mathbb{V}_{\mathcal{Q}}, \mathcal{L}\right)$, where the entries of $\mathcal{L}$ are meromorphic functions on $\mathbb{C}$ with values in $\operatorname{End}\left(\mathbb{V}_{\mathcal{Q}}\right)$.

\section{B. Modules over $\mathscr{A}(\mathcal{R})$}

Let $\mathcal{L}_{k}: \mathbb{C} \rightarrow \operatorname{End}\left(\mathbb{V}_{k} \otimes \mathbb{V}_{\mathcal{Q}}\right)$ for $k=i, j$ be meromorphic and recall that $\mathbb{V}_{\mathcal{Q}}$ introduced in Sec. II A is a diagonalizable module. Then, according to the Yang-Baxter equation (2.12), the pair $\left(\mathbb{V}_{\mathcal{Q}}, \mathcal{L}\right)$ fulfills (3.1) in $\operatorname{End}\left(\mathbb{V}_{i} \otimes \mathbb{V}_{j} \otimes \mathbb{V}_{\mathcal{Q}}\right)$ with $\mathcal{L}$ identified with the monodromy matrix $\mathcal{T}$ defined in $(2.7)$.

\section{Singular vectors}

Next, we shall describe a class of singular vectors in the $\mathscr{A}(\mathcal{R})$-module $\left(\mathbb{V}_{\mathcal{Q}}, \mathcal{L}\right)$. More precisely, we refer to singular vectors as the nonzero elements $v_{0} \in \mathbb{V}_{\mathcal{Q}}$ such that $\mathcal{C}_{i}(\lambda) v_{0}=0(i=1,2,3)$ for all $\lambda \in \mathbb{C}$, with (2.8) taken into account. In addition to that, we assign the weight $\left(\Lambda_{1}(\lambda), \Lambda_{2}(\lambda), \Lambda_{3}(\lambda)\right)$ to an element $v \in \mathbb{V}_{\mathcal{Q}}$ satisfying $\mathcal{A}_{i}(\lambda) v=\Lambda_{i}(\lambda) v$ for $i=1,2,3$. The above definitions, together with the $\mathscr{A}(\mathscr{R})$-module discussed in Sec. III B, pave the way to introduce a weight-module constituted of singular vectors $v_{0} \in \mathbb{V}_{\mathcal{Q}}$ with weight $\left(\Lambda_{1}(\lambda), \Lambda_{2}(\lambda), \Lambda_{3}(\lambda)\right.$ ). Hence, according to (2.7) and (2.8), one can show that the vector $|0\rangle$ being previously defined in Sec. II C belongs to the above-defined weight-module with weights

$$
\Lambda_{1}(\lambda):=\prod_{j=1}^{L} a\left(\lambda-\mu_{j}\right), \quad \Lambda_{2}(\lambda):=\prod_{j=1}^{L} b\left(\lambda-\mu_{j}\right), \quad \text { and } \quad \Lambda_{3}(\lambda):=\prod_{j=1}^{L} d_{3,3}\left(\lambda-\mu_{j}\right) .
$$

Dual singular vectors and dual weight-modules are defined in a similar way. For instance, we call dual singular vectors the elements $v_{0}^{\dagger} \in \mathbb{V}_{\mathcal{Q}}^{\dagger}$ such that $v_{0}^{\dagger} \mathcal{C}_{i}(\lambda)=0(i=1,2,3)$ for all $\lambda \in \mathbb{C}$. Moreover, in order to characterize a dual weight-module, we assign the weight $\left(\bar{\Lambda}_{1}(\lambda), \bar{\Lambda}_{2}(\lambda), \bar{\Lambda}_{3}(\lambda)\right)$ to any element $v^{\dagger} \in \mathbb{V}_{\mathcal{Q}}^{\dagger}$ satisfying $v^{\dagger} \mathcal{A}_{i}(\lambda)=\bar{\Lambda}_{i}(\lambda) v^{\dagger}$ for $i=1,2,3$. In this way, we define a dual weight-module consisting of dual singular vectors with the aforementioned weight. It is then clear that the dual vector $\langle\overline{0}|$ defined in Sec. II C belongs to the dual weight-module with weights

$$
\bar{\Lambda}_{1}(\lambda):=\prod_{j=1}^{L} d_{1,1}\left(\lambda-\mu_{j}\right), \quad \bar{\Lambda}_{2}(\lambda):=\prod_{j=1}^{L} b\left(\lambda-\mu_{j}\right), \quad \text { and } \quad \bar{\Lambda}_{3}(\lambda):=\prod_{j=1}^{L} a_{3,3}\left(\lambda-\mu_{j}\right) .
$$

\section{Higher-order relations}

The most notable use of the algebra $\mathscr{A}(\mathscr{R})$ can be found within the context of the Algebraic Bethe Ansatz (ABA) method ${ }^{37,38}$ and Sklyanin's separation of variables. ${ }^{39}$ Both methods have been used for the diagonalization of transfer matrices associated with integrable vertex models with periodic boundary conditions and variations. The AF method can then be regarded as an alternative use of the algebra $\mathscr{A}(\mathscr{R})$, and it has found fruitful soil in models with domain-wall boundary conditions. The ABA, in its turn, has been formulated for a variety of models, including nineteen-vertex models, ${ }^{7}$ and it is then natural to speculate if the AF method can also be applied to nineteen-vertex models 
with domain-wall boundaries. In order to investigate such possibility, it is important to first examine the particular algebraic relations in $\mathscr{A}(\mathscr{R})$ associated with nineteen-vertex models. In that case, $\mathscr{A}(\mathscr{R})$ is an algebra over $\mathbb{C}$ generated by elements $\mathcal{A}_{i}, \mathcal{B}_{i}$, and $\mathcal{C}_{i}$ defined in (2.8). In particular, $\mathscr{A}(\mathscr{R})$ will be regarded as a matrix algebra with elements in $\mathbb{C}\left[\left[x, x^{-1}\right]\right] \otimes \operatorname{End}\left(\mathbb{V}_{\mathcal{Q}}\right)$. We shall also use $\mathscr{A}_{2}(\mathcal{R})$ to denote the Yang-Baxter algebra $\mathscr{A}(\mathscr{R})$ in order to emphasize that it is a quadratic algebra. Next, we introduce $\mathscr{M}_{n}:=\left\{\mathcal{A}_{i}, \mathcal{B}_{i}, \mathcal{C}_{i} \mid i=1,2,3\right\}\left(x_{n-1}\right)$ such that one can define $\mathscr{A}_{n}(\mathcal{R}) \simeq \mathscr{A}_{n-1}(\mathcal{R}) \otimes \mathscr{M}_{n} / \mathscr{A}_{2}(\mathcal{R})$ for $n>2$ through the repeated use of $\mathscr{A}(\mathscr{R})$. We refer to $\mathscr{A}_{n}(\mathcal{R})$ as a higherorder Yang-Baxter algebra, and in what follows, we shall look for relations in $\mathscr{A}_{n}(\mathcal{R})$ suitable for the implementation of the AF method. For instance, the elimination of terms of the form $\mathcal{E}(\lambda) \mathcal{A}(\mu)$ using the first relation in (A3) and the second relation in (A2) gives us the following relation in $\mathscr{A}_{2}(\mathcal{R})$ :

$$
\begin{aligned}
{\left[\frac{d_{1,1}\left(\lambda_{1}-\lambda_{0}\right)}{d_{1,2}\left(\lambda_{1}-\lambda_{0}\right)}-\frac{d_{3,1}\left(\lambda_{1}-\lambda_{0}\right)}{d_{3,2}\left(\lambda_{1}-\lambda_{0}\right)}\right] \mathcal{A}\left(\lambda_{0}\right) \mathcal{E}\left(\lambda_{1}\right)+\frac{a\left(\lambda_{1}-\lambda_{0}\right)}{d_{3,2}\left(\lambda_{1}-\lambda_{0}\right)} \mathcal{A}\left(\lambda_{1}\right) \mathcal{E}\left(\lambda_{0}\right)=} & {\left[\frac{d_{2,1}\left(\lambda_{1}-\lambda_{0}\right)}{d_{3,2}\left(\lambda_{1}-\lambda_{0}\right)}-\frac{d_{2,2}\left(\lambda_{1}-\lambda_{0}\right)}{d_{3,2}\left(\lambda_{1}-\lambda_{0}\right)} \frac{d_{1,1}\left(\lambda_{1}-\lambda_{0}\right)}{d_{1,2}\left(\lambda_{1}-\lambda_{0}\right)}\right] \mathcal{B}\left(\lambda_{0}\right) \mathcal{B}\left(\lambda_{1}\right) } \\
& +\frac{a\left(\lambda_{1}-\lambda_{0}\right)}{d_{3,2}\left(\lambda_{1}-\lambda_{0}\right)} \frac{d_{1,1}\left(\lambda_{1}-\lambda_{0}\right)}{d_{1,2}\left(\lambda_{1}-\lambda_{0}\right)} \mathcal{B}\left(\lambda_{1}\right) \mathcal{B}\left(\lambda_{0}\right) .
\end{aligned}
$$

Remark 3.1. Another relation exhibiting the same structure of (3.4) but with apparently different coefficients can also be obtained by combining the first relation in (A3) and the third relation in (A1). However, a closer look at the coefficients of this alternative relation shows that it is not linearly independent from (3.4).

Along the same lines employed in the derivation of (3.4), we also notice that the elimination of $\mathcal{A}(\lambda) \mathcal{E}(\mu)$ in between the first relation in (A3) and the third relation in (A1) leaves us with the relation

$$
\begin{gathered}
\frac{a\left(\lambda_{1}-\lambda_{0}\right)}{d_{3,3}\left(\lambda_{1}-\lambda_{0}\right)} \mathcal{E}\left(\lambda_{1}\right) \mathcal{A}\left(\lambda_{0}\right)+\left[\frac{d_{1,2}\left(\lambda_{1}-\lambda_{0}\right)}{d_{3,2}\left(\lambda_{1}-\lambda_{0}\right)}-\frac{d_{1,3}\left(\lambda_{1}-\lambda_{0}\right)}{d_{3,3}\left(\lambda_{1}-\lambda_{0}\right)}\right] \mathcal{E}\left(\lambda_{0}\right) \mathcal{A}\left(\lambda_{1}\right)= \\
\frac{a\left(\lambda_{1}-\lambda_{0}\right)}{d_{3,2}\left(\lambda_{1}-\lambda_{0}\right)} \mathcal{B}\left(\lambda_{1}\right) \mathcal{B}\left(\lambda_{0}\right)+\left[\frac{d_{2,3}\left(\lambda_{1}-\lambda_{0}\right)}{d_{3,3}\left(\lambda_{1}-\lambda_{0}\right)}-\frac{d_{2,2}\left(\lambda_{1}-\lambda_{0}\right)}{d_{3,2}\left(\lambda_{1}-\lambda_{0}\right)}\right] \mathcal{B}\left(\lambda_{0}\right) \mathcal{B}\left(\lambda_{1}\right) .
\end{gathered}
$$

Remark 3.2. The elimination of $\mathcal{A}(\lambda) \mathcal{E}(\mu)$ using the first relation in (A3) and the second relation in (A2) also yields a relation with the same structure of (3.5). However, similarly to Remark 3.1, the resulting relation is not linearly independent from (3.5).

Relations (3.4) and (3.5) both live in $\mathscr{A}_{2}(\mathcal{R})$, and, as it will become clear later on, we shall need relations in $\mathscr{A}_{L+1}(\mathcal{R})$ which can be exploited along the AF method. Such relations can then be obtained by letting (3.4) to act on $\prod_{2 \leq j \leq L} \mathcal{E}\left(\lambda_{j}\right)$ from the left and by letting (3.5) to act on the same product of operators from the right. In this way, considering the third relation in (A3), we are left with the following relations in $\mathscr{A}_{L+1}(\mathcal{R})$ :

$$
\begin{aligned}
& {\left[\frac{d_{1,1}\left(\lambda_{1}-\lambda_{0}\right)}{d_{1,2}\left(\lambda_{1}-\lambda_{0}\right)}-\frac{d_{3,1}\left(\lambda_{1}-\lambda_{0}\right)}{d_{3,2}\left(\lambda_{1}-\lambda_{0}\right)}\right] \mathcal{A}\left(\lambda_{0}\right) \prod_{\substack{0 \leq j \leq L \\
j \neq 0}}^{\rightarrow} \mathcal{E}\left(\lambda_{j}\right)+\frac{a\left(\lambda_{1}-\lambda_{0}\right)}{d_{3,2}\left(\lambda_{1}-\lambda_{0}\right)} \mathcal{A}\left(\lambda_{1}\right) \prod_{\substack{0 \leq j \leq L \\
j \neq 0}}^{\rightarrow} \mathcal{E}\left(\lambda_{j}\right)=} \\
& {\left[\frac{d_{2,1}\left(\lambda_{1}-\lambda_{0}\right)}{d_{3,2}\left(\lambda_{1}-\lambda_{0}\right)}-\frac{d_{2,2}\left(\lambda_{1}-\lambda_{0}\right)}{d_{3,2}\left(\lambda_{1}-\lambda_{0}\right)} \frac{d_{1,1}\left(\lambda_{1}-\lambda_{0}\right)}{d_{1,2}\left(\lambda_{1}-\lambda_{0}\right)}\right] \mathcal{B}\left(\lambda_{0}\right) \mathcal{B}\left(\lambda_{1}\right) \prod_{2 \leq j \leq L}^{\rightarrow} \mathcal{E}\left(\lambda_{j}\right)} \\
& +\frac{a\left(\lambda_{1}-\lambda_{0}\right)}{d_{3,2}\left(\lambda_{1}-\lambda_{0}\right)} \frac{d_{1,1}\left(\lambda_{1}-\lambda_{0}\right)}{d_{1,2}\left(\lambda_{1}-\lambda_{0}\right)} \mathcal{B}\left(\lambda_{1}\right) \mathcal{B}\left(\lambda_{0}\right) \prod_{2 \leq j \leq L}^{\rightarrow} \mathcal{E}\left(\lambda_{j}\right) \\
& \frac{a\left(\lambda_{1}-\lambda_{0}\right)}{d_{3,3}\left(\lambda_{1}-\lambda_{0}\right)} \prod_{\substack{0 \leq j \leq L \\
j \neq 0}}^{\rightarrow} \mathcal{E}\left(\lambda_{j}\right) \mathcal{A}\left(\lambda_{0}\right)+\left[\frac{d_{1,2}\left(\lambda_{1}-\lambda_{0}\right)}{d_{3,2}\left(\lambda_{1}-\lambda_{0}\right)}-\frac{d_{1,3}\left(\lambda_{1}-\lambda_{0}\right)}{d_{3,3}\left(\lambda_{1}-\lambda_{0}\right)}\right] \prod_{\substack{0 \leq j \leq L \\
j \neq 1}}^{\rightarrow} \mathcal{E}\left(\lambda_{j}\right) \mathcal{A}\left(\lambda_{1}\right)= \\
& {\left[\frac{d_{2,3}\left(\lambda_{1}-\lambda_{0}\right)}{d_{3,3}\left(\lambda_{1}-\lambda_{0}\right)}-\frac{d_{2,2}\left(\lambda_{1}-\lambda_{0}\right)}{d_{3,2}\left(\lambda_{1}-\lambda_{0}\right)}\right] \prod_{2 \leq j \leq L}^{\rightarrow} \mathcal{E}\left(\lambda_{j}\right) \mathcal{B}\left(\lambda_{0}\right) \mathcal{B}\left(\lambda_{1}\right)} \\
& +\frac{a\left(\lambda_{1}-\lambda_{0}\right)}{d_{3,2}\left(\lambda_{1}-\lambda_{0}\right)} \prod_{2 \leq j \leq L}^{\rightarrow} \mathcal{E}\left(\lambda_{j}\right) \mathcal{B}\left(\lambda_{1}\right) \mathcal{B}\left(\lambda_{0}\right)
\end{aligned}
$$


Now one can readily recognize the product of operators appearing in the RHS of (3.6) and (3.7) as the same operators characterizing the partition functions $\mathcal{F}$ and $\overline{\mathcal{F}}$ according to (2.19). As it will become clear later on, we then proceed by looking for relations in $\mathscr{A}_{L+1}(\mathcal{R})$ allowing us to express $\mathcal{B}(\lambda) \mathcal{B}(\mu) \underset{2 \leq j \leq L}{\prod_{1}} \mathcal{E}\left(\lambda_{j}\right)$ in terms of $\prod_{2 \leq j \leq L} \mathcal{E}\left(\lambda_{j}\right) \mathcal{B}(\bar{\lambda}) \mathcal{B}(\bar{\mu})$ and vice versa. Such a task can be accomplished through the use of suitable commutation relations in $\mathscr{S}_{\mathcal{A}, \mathcal{B}, \mathcal{E}}$. For instance, we shall use the third relation in (A2) and the last two relations in (A3). Such commutation rules read

$$
\begin{aligned}
\mathcal{B}\left(\lambda_{0}\right) \mathcal{E}\left(\lambda_{1}\right) & =\frac{a\left(\lambda_{1}-\lambda_{0}\right)}{b\left(\lambda_{1}-\lambda_{0}\right)} \mathcal{E}\left(\lambda_{1}\right) \mathcal{B}\left(\lambda_{0}\right)-\frac{c\left(\lambda_{1}-\lambda_{0}\right)}{b\left(\lambda_{1}-\lambda_{0}\right)} \mathcal{E}\left(\lambda_{0}\right) \mathcal{B}\left(\lambda_{1}\right), \\
\mathcal{E}\left(\lambda_{0}\right) \mathcal{B}\left(\lambda_{1}\right) & =\frac{a\left(\lambda_{1}-\lambda_{0}\right)}{b\left(\lambda_{1}-\lambda_{0}\right)} \mathcal{B}\left(\lambda_{1}\right) \mathcal{E}\left(\lambda_{0}\right)-\frac{\bar{c}\left(\lambda_{1}-\lambda_{0}\right)}{b\left(\lambda_{1}-\lambda_{0}\right)} \mathcal{B}\left(\lambda_{0}\right) \mathcal{E}\left(\lambda_{1}\right), \\
\mathcal{E}\left(\lambda_{0}\right) \mathcal{E}\left(\lambda_{1}\right) & =\mathcal{E}\left(\lambda_{1}\right) \mathcal{E}\left(\lambda_{0}\right),
\end{aligned}
$$

and they provide neat exchange relations between the operators $\mathcal{B}$ and $\mathcal{E}$. Moreover, one can notice that (3.8) are essentially the same commutation relations found in the six-vertex model. ${ }^{40}$ Therefore, we can readily use the known results for the six-vertex model to find the following relations in $\mathscr{A}_{n+2}(\mathcal{R})$ :

$$
\begin{aligned}
& \mathcal{B}\left(\lambda_{n+1}\right) \mathcal{B}\left(\lambda_{0}\right) \prod_{1 \leq j \leq n} \mathcal{E}\left(\lambda_{j}\right)=\sum_{j=0}^{n} \sum_{\substack{k=0 \\
k \neq j}}^{n+1} \mathcal{M}_{j}^{(n)} \mathcal{N}_{j, k}^{(n)} \prod_{\substack{0 \leq l \leq n+1 \\
l \neq j, k}}^{\rightarrow} \mathcal{E}\left(\lambda_{l}\right) \mathcal{B}\left(\lambda_{k}\right) \mathcal{B}\left(\lambda_{j}\right), \\
& \prod_{1 \leq j \leq n} \mathcal{E}\left(\lambda_{j}\right) \mathcal{B}\left(\lambda_{0}\right) \mathcal{B}\left(\lambda_{n+1}\right)=\sum_{j=0}^{n} \sum_{\substack{k=0 \\
k \neq j}}^{n+1} \overline{\mathcal{M}}_{j}^{(n)} \overline{\mathcal{N}}_{j, k}^{(n)} \mathcal{B}\left(\lambda_{j}\right) \mathcal{B}\left(\lambda_{k}\right) \prod_{\substack{0 \leq l \leq n+1 \\
l \neq j, k}}^{\rightarrow} \mathcal{E}\left(\lambda_{l}\right) .
\end{aligned}
$$

The coefficients in (3.9) are in their turn given by

$$
\begin{aligned}
& \mathcal{M}_{j}^{(n)}:=\left\{\begin{array}{lc}
\prod_{l=1}^{n} \frac{a\left(\lambda_{l}-\lambda_{0}\right)}{b\left(\lambda_{l}-\lambda_{0}\right)}, & j=0, \\
-\frac{c\left(\lambda_{j}-\lambda_{0}\right)}{b\left(\lambda_{j}-\lambda_{0}\right)} \prod_{l=1}^{n} \frac{a\left(\lambda_{l}-\lambda_{j}\right)}{b\left(\lambda_{l}-\lambda_{j}\right)}, & 1 \leq j \leq n,
\end{array}\right. \\
& \mathcal{N}_{j, k}^{(n)}:=\left\{\begin{array}{lr}
\prod_{l=0}^{n} \frac{a\left(\lambda_{l}-\lambda_{n+1}\right)}{b\left(\lambda_{l}-\lambda_{n+1}\right)}, & k=n+1, \\
l \neq j & 0 \leq k \leq n ; k \neq j, \\
-\frac{c\left(\lambda_{k}-\lambda_{n+1}\right)}{b\left(\lambda_{k}-\lambda_{n+1}\right)} \prod_{l=0}^{n} \frac{a\left(\lambda_{l}-\lambda_{k}\right)}{b\left(\lambda_{l}-\lambda_{k}\right)}, &
\end{array}\right.
\end{aligned}
$$

while the ones in (3.10) read

$$
\begin{aligned}
& \overline{\mathcal{M}}_{j}^{(n)}:=\left\{\begin{array}{lc}
\prod_{l=1}^{n} \frac{a\left(\lambda_{0}-\lambda_{l}\right)}{b\left(\lambda_{0}-\lambda_{l}\right)}, & j=0, \\
-\frac{\bar{c}\left(\lambda_{0}-\lambda_{j}\right)}{b\left(\lambda_{0}-\lambda_{j}\right)} \prod_{\substack{l=1 \\
l \neq j}}^{n} \frac{a\left(\lambda_{j}-\lambda_{l}\right)}{b\left(\lambda_{j}-\lambda_{l}\right)}, & 1 \leq j \leq n,
\end{array}\right.
\end{aligned}
$$

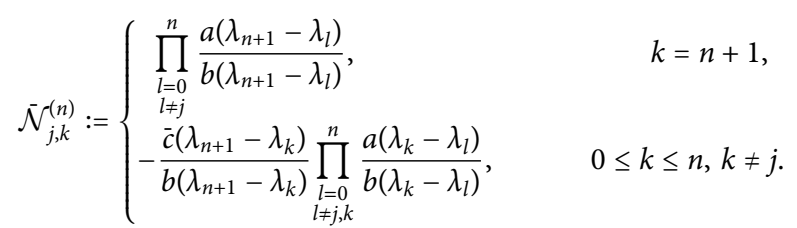

As previously remarked, our approach will require relations of types (3.9) and (3.10) in $\mathscr{A}_{L+1}(\mathcal{R})$. The latter can then be obtained from (3.9) and (3.10) by setting $n=L-1$. 


\section{E. The functional $\Phi$}

After having established suitable higher-order algebraic relations in $\mathscr{A}_{n+1}(\mathcal{R})$, the next step within the AF approach is to find a linear functional $\Phi: \mathscr{A}_{n+1}(\mathcal{R}) \rightarrow \mathbb{C}\left[\lambda_{0}^{ \pm 1}, \lambda_{1}^{ \pm 1}, \ldots, \lambda_{n}^{ \pm 1}\right]$ allowing us to write functional equations for quantities of interest. In particular, we would like the functional $\Phi$ to satisfy the property

$$
\Phi\left(J_{n}\right)=\omega_{J}\left(\lambda_{0}, \lambda_{1}, \ldots, \lambda_{n-1}\right) \Phi\left(J_{n-1}\right)
$$

for certain elements $J_{n} \subseteq \mathscr{A}_{n}(\mathcal{R})$ and a fixed meromorphic functions $\omega_{J}$. As for the characterization of the partition functions $\mathcal{Z}, \mathcal{F}$, and $\overline{\mathcal{F}}$, we shall employ the higher-order relations (3.6), (3.7), (3.9), and (3.10) in $\mathscr{A}_{L+1}(\mathcal{R})$. Moreover, a closer inspection of such relations suggests considering the following realization of the functional $\Phi$, namely,

$$
\Phi\left(J_{L+1}\right)=\left\langle\overline{0}\left|J_{L+1}\right| 0\right\rangle
$$

with vectors $\langle\overline{0}|$ and $|0\rangle$ being previously defined in Sec. II C. In Sec. IV, we shall then precise the functional equations obtained from the application of (3.14) on (3.6), (3.7), (3.9), and (3.10).

\section{FUNCTIONAL EQUATIONS}

This section is concerned with the explicit construction and analysis of functional relations satisfied by the partition functions $\mathcal{Z}, \mathcal{F}$, and $\overline{\mathcal{F}}$, using the AF method described in Sec. III. However, it is fair to say that in Sec. III, we have only collected the ingredients required for the derivation of the anticipated functional equations. Here, we intend to bring that procedure to conclusion by combining all those ingredients in a suitable way. For that, it is convenient to introduce the following extra conventions.

Let us write $\mathrm{X}:=\left\{\lambda_{1}, \lambda_{2}, \ldots, \lambda_{L}\right\}$ for fixed $L \in \mathbb{Z}_{\leq 1}$ and additionally introduce the short-hand notation

$$
\mathrm{X}_{\alpha_{1}, \alpha_{2}, \ldots, \alpha_{l}}^{\beta_{1}, \beta_{2}, \ldots, \beta_{m}}:=\mathrm{X} \cup\left\{\lambda_{\beta_{1}}, \lambda_{\beta_{2}}, \ldots, \lambda_{\beta_{m}}\right\} \backslash\left\{\lambda_{\alpha_{1}}, \lambda_{\alpha_{2}}, \ldots, \lambda_{\alpha_{l}}\right\}
$$

Moreover, we shall also use

$$
\begin{array}{ll}
\Lambda(\lambda):=\prod_{j=1}^{L} a\left(\lambda-\mu_{j}\right), & \bar{\Lambda}(\lambda):=\prod_{j=1}^{L} d_{1,1}\left(\lambda-\mu_{j}\right), \\
\omega(\lambda):=\prod_{j=1}^{L}\left(e^{2 \lambda}-e^{2 \mu_{j}} \zeta\right), & \bar{\omega}(\lambda):=\prod_{j=1}^{L}\left(e^{2 \lambda}-e^{2 \mu_{j}}\right) .
\end{array}
$$

In this way, we can construct the following functional relations.

Lemma 4.1. The functions $\mathcal{Z}$ and $\mathcal{H}$ are related through the equation

$$
\Omega_{0} \mathcal{Z}(\mathrm{X})+\Omega_{1} \mathcal{Z}\left(\mathrm{X}_{1}^{0}\right)=\Upsilon_{0} \mathcal{H}\left(\mathrm{X}_{1} \mid \lambda_{0}, \lambda_{1}\right)+\Upsilon_{1} \mathcal{H}\left(\mathrm{X}_{1} \mid \lambda_{1}, \lambda_{0}\right),
$$

with coefficients

$$
\begin{array}{ll}
\Omega_{0}:=\frac{a\left(\lambda_{1}-\lambda_{0}\right)}{d_{3,3}\left(\lambda_{1}-\lambda_{0}\right)} \Lambda\left(\lambda_{0}\right), & \Omega_{1}:=\left[\frac{d_{1,2}\left(\lambda_{1}-\lambda_{0}\right)}{d_{3,2}\left(\lambda_{1}-\lambda_{0}\right)}-\frac{d_{1,3}\left(\lambda_{1}-\lambda_{0}\right)}{d_{3,3}\left(\lambda_{1}-\lambda_{0}\right)}\right] \Lambda\left(\lambda_{1}\right), \\
\Upsilon_{0}:=\frac{a\left(\lambda_{1}-\lambda_{0}\right)}{d_{3,2}\left(\lambda_{1}-\lambda_{0}\right)} \omega\left(\lambda_{0}\right), & \Upsilon_{1}:=\left[\frac{d_{2,3}\left(\lambda_{1}-\lambda_{0}\right)}{d_{3,3}\left(\lambda_{1}-\lambda_{0}\right)}-\frac{d_{2,2}\left(\lambda_{1}-\lambda_{0}\right)}{d_{3,2}\left(\lambda_{1}-\lambda_{0}\right)}\right] \omega\left(\lambda_{1}\right) .
\end{array}
$$

Proof. The proof is straightforward, and Eq. (4.3) follows from the application of the functional $\Phi$ defined in (3.14) on the higher-order relation (3.7). In addition, in order to obtain (4.3), one also needs to recall that $|0\rangle$ and $|\overline{0}\rangle$ are singular vectors, with properties described in Sec. III C, and use formulas (2.19) and (2.29).

Lemma 4.2. Similarly to Lemma 4.1, there also exists a functional relation between $\mathcal{Z}$ and $\overline{\mathcal{H}}$, namely,

$$
\bar{\Omega}_{0} \mathcal{Z}(\mathrm{X})+\bar{\Omega}_{1} \mathcal{Z}\left(\mathrm{X}_{1}^{0}\right)=\bar{\Upsilon}_{0} \overline{\mathcal{H}}\left(\lambda_{1}, \lambda_{0} \mid \mathrm{X}_{1}\right)+\bar{\Upsilon}_{1} \overline{\mathcal{H}}\left(\lambda_{0}, \lambda_{1} \mid \mathrm{X}_{1}\right)
$$

with coefficients reading

$$
\begin{aligned}
& \bar{\Omega}_{0}:=\left[\frac{d_{1,1}\left(\lambda_{1}-\lambda_{0}\right)}{d_{1,2}\left(\lambda_{1}-\lambda_{0}\right)}-\frac{d_{3,1}\left(\lambda_{1}-\lambda_{0}\right)}{d_{3,2}\left(\lambda_{1}-\lambda_{0}\right)}\right] \bar{\Lambda}\left(\lambda_{0}\right), \quad \bar{\Upsilon}_{1}:=\frac{a\left(\lambda_{1}-\lambda_{0}\right)}{d_{3,2}\left(\lambda_{1}-\lambda_{0}\right)} \frac{d_{1,1}\left(\lambda_{1}-\lambda_{0}\right)}{d_{1,2}\left(\lambda_{1}-\lambda_{0}\right)} \bar{\omega}\left(\lambda_{1}\right), \\
& \bar{\Upsilon}_{0}:=\left[\frac{d_{2,1}\left(\lambda_{1}-\lambda_{0}\right)}{d_{3,2}\left(\lambda_{1}-\lambda_{0}\right)}-\frac{d_{2,2}\left(\lambda_{1}-\lambda_{0}\right)}{d_{3,2}\left(\lambda_{1}-\lambda_{0}\right)} \frac{d_{1,1}\left(\lambda_{1}-\lambda_{0}\right)}{d_{1,2}\left(\lambda_{1}-\lambda_{0}\right)}\right] \bar{\omega}\left(\lambda_{0}\right), \quad \bar{\Omega}_{1}:=\frac{a\left(\lambda_{1}-\lambda_{0}\right)}{d_{3,2}\left(\lambda_{1}-\lambda_{0}\right)} \bar{\Lambda}\left(\lambda_{1}\right) .
\end{aligned}
$$


Proof. Along the same lines used in the proof of Lemma 4.1, we simply apply the functional $\Phi$ on the higher-order relation (3.6), keeping in mind formulas (2.19) and (2.31).

Some comments are in order at this point. For instance, Lemma 4.1 establishes a relation between a set of functions $\mathcal{Z}$ and a set of functions $\mathcal{H}$. In this way, once the function $\mathcal{H}$ is known, we would have a functional equation involving solely the partition function $\mathcal{Z}$. This would be the optimal situation resembling the equations found in the six-vertex model through the AF method. In addition, we stress here that the same remarks apply when considering Lemma 4.2 and the function $\overline{\mathcal{H}}$. However, we are in the situation that neither $\mathcal{H}$ nor $\overline{\mathcal{H}}$ is known a priori, and this fact makes our analysis significantly more involved. Therefore, in order to circumvent the aforementioned difficulty, we shall consider the following strategy. Taking into account the functional relations described in Lemmas 4.1 and 4.2 , one can notice that we would have an effective relation between functions $\mathcal{Z}$ (with different spectral parameters) in case we were able to relate the functions $\mathcal{H}$ and $\overline{\mathcal{H}}$ appearing, respectively, in Lemmas 4.1 and 4.2. In this way, one could regard $\mathcal{H}$ and $\overline{\mathcal{H}}$ as auxiliary functions, and a schematic representation of this strategy is shown in Fig. 10. Fortunately, the sought relation between $\mathcal{H}$ and $\overline{\mathcal{H}}$ can also be obtained using the AF method and we shall refer to the resulting system of equations as the $\mathrm{ZH}$-system.

Lemma 4.3. The following relations hold:

$$
\begin{aligned}
& \omega\left(\lambda_{L}\right) \mathcal{H}\left(\mathrm{X}_{L} \mid \lambda_{L}, \lambda_{0}\right)=\sum_{j=0}^{L-1} \sum_{\substack{k=0 \\
k \neq j}}^{L} \overline{\mathcal{M}}_{j}^{(L-1)} \overline{\mathcal{N}}_{j, k}^{(L-1)} \bar{\omega}\left(\lambda_{j}\right) \overline{\mathcal{H}}\left(\lambda_{k}, \lambda_{j} \mid \mathrm{X}_{j, k}^{0}\right), \\
& \bar{\omega}\left(\lambda_{L}\right) \overline{\mathcal{H}}\left(\lambda_{0}, \lambda_{L} \mid \mathrm{X}_{L}\right)=\sum_{j=0}^{L-1} \sum_{\substack{k=0 \\
k \neq j}}^{L} \mathcal{M}_{j}^{(L-1)} \mathcal{N}_{j, k}^{(L-1)} \omega\left(\lambda_{j}\right) \mathcal{H}\left(\mathrm{X}_{j, k}^{0} \mid \lambda_{j}, \lambda_{k}\right) .
\end{aligned}
$$

Proof. We apply the functional $\Phi$ defined in (3.14) on the higher-order relations (3.9) with $n=L-1$. Then, we are able to recognize the functions $\mathcal{H}$ and $\mathcal{H}$ with the help of (2.19), (2.29), and (2.31).

Remark 4.4. The inspection of Eq. (4.7) for small values of $L$ shows that the first equation is immediately satisfied upon the substitution of the second equation and vice versa. Therefore, Eq. (4.7) are not linearly independent and one can consider only one of them.

\section{A. The ZH-system}

Lemmas 4.1-4.3 describe a system of functional equations relating the partition functions with domain-wall boundaries discussed in Sec. II C. Here, we shall refer to the above-described system of equations as the ZH-system and, more precisely, it comprises Eqs. (4.3), (4.5), and (4.7). Our partition functions $\mathcal{Z}, \mathcal{F}$, and $\overline{\mathcal{F}}$ satisfy the $\mathrm{ZH}$-system by construction; however, it is not $a$ priori clear if the $\mathrm{ZH}$-system fixes uniquely such functions. Here, we claim this is indeed the case, and to support our claim, we proceed with a more detailed analysis of the $\mathrm{ZH}$-system and present explicit solutions for small lattices.

We start our analysis by recalling that the coefficients given in (4.4) and (4.6) depend solely on the spectral parameters $\lambda_{0}$ and $\lambda_{1}$. Hence, in order to emphasize such dependence, we also write $\Omega_{i}=\Omega_{i}\left(\lambda_{0}, \lambda_{1}\right), \bar{\Omega}_{i}=\bar{\Omega}_{i}\left(\lambda_{0}, \lambda_{1}\right), \Upsilon_{i}=\Upsilon_{i}\left(\lambda_{0}, \lambda_{1}\right)$, and $\bar{\Upsilon}_{i}=\bar{\Upsilon}_{i}\left(\lambda_{0}, \lambda_{1}\right)$. Next, we use Cramer's method to solve the system of equations formed by (4.3) and (4.5) for $\mathcal{Z}(\mathrm{X})$ and $\mathcal{Z}\left(\mathrm{X}_{1}^{0}\right)$. By doing so, we obtain two expressions for the function $\mathcal{Z}$, one depending on the set of variables $\mathrm{X}$ and another one for $\mathcal{Z}$ depending on $\mathrm{X}_{1}^{0}$. The obtained expressions need to be consistent and denote the same function upon an appropriate renaming of variables. In this way, we are left with a functional relation between $\mathcal{H}$ and $\overline{\mathcal{H}}$. More precisely, the resolution of (4.3) and (4.5) yields the expressions

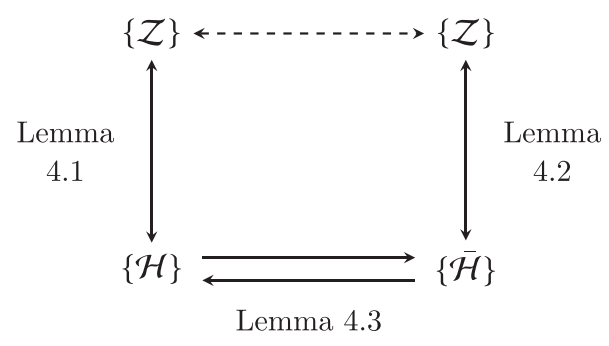

FIG. 10. Schematic representation of the ZH-system. 


$$
\begin{aligned}
\mathcal{Z}(\mathrm{X})= & \frac{\Upsilon_{0}\left(\lambda_{0}, \lambda_{1}\right) \bar{\Omega}_{1}\left(\lambda_{0}, \lambda_{1}\right)}{\mathcal{W}\left(\lambda_{0}, \lambda_{1}\right)} \mathcal{H}\left(\mathrm{X}_{1} \mid \lambda_{0}, \lambda_{1}\right)+\frac{\Upsilon_{1}\left(\lambda_{0}, \lambda_{1}\right) \bar{\Omega}_{1}\left(\lambda_{0}, \lambda_{1}\right)}{\mathcal{W}\left(\lambda_{0}, \lambda_{1}\right)} \mathcal{H}\left(\mathrm{X}_{1} \mid \lambda_{1}, \lambda_{0}\right) \\
& -\frac{\bar{\Upsilon}_{0}\left(\lambda_{0}, \lambda_{1}\right) \Omega_{1}\left(\lambda_{0}, \lambda_{1}\right)}{\mathcal{W}\left(\lambda_{0}, \lambda_{1}\right)} \overline{\mathcal{H}}\left(\lambda_{1}, \lambda_{0} \mid \mathrm{X}_{1}\right)-\frac{\bar{\Upsilon}_{1}\left(\lambda_{0}, \lambda_{1}\right) \Omega_{1}\left(\lambda_{0}, \lambda_{1}\right)}{\mathcal{W}\left(\lambda_{0}, \lambda_{1}\right)} \overline{\mathcal{H}}\left(\lambda_{0}, \lambda_{1} \mid \mathrm{X}_{1}\right)
\end{aligned}
$$

and

$$
\begin{aligned}
\mathcal{Z}(\mathrm{X})= & \frac{\Omega_{0}\left(\lambda_{1}, \lambda_{\overline{0}}\right) \bar{\Upsilon}_{1}\left(\lambda_{1}, \lambda_{\overline{0}}\right)}{\mathcal{W}\left(\lambda_{1}, \lambda_{\overline{0}}\right)} \overline{\mathcal{H}}\left(\lambda_{1}, \lambda_{\overline{0}} \mid \mathrm{X}_{1}\right)+\frac{\Omega_{0}\left(\lambda_{1}, \lambda_{\overline{0}}\right) \bar{\Upsilon}_{0}\left(\lambda_{1}, \lambda_{\overline{0}}\right)}{\mathcal{W}\left(\lambda_{1}, \lambda_{\overline{0}}\right)} \overline{\mathcal{H}}\left(\lambda_{\overline{0}}, \lambda_{1} \mid \mathrm{X}_{1}\right) \\
& -\frac{\bar{\Omega}_{0}\left(\lambda_{1}, \lambda_{\overline{0}}\right) \Upsilon_{1}\left(\lambda_{1}, \lambda_{\overline{0}}\right)}{\mathcal{W}\left(\lambda_{1}, \lambda_{\overline{0}}\right)} \mathcal{H}\left(\mathrm{X}_{1} \mid \lambda_{\overline{0}}, \lambda_{1}\right)-\frac{\bar{\Omega}_{0}\left(\lambda_{1}, \lambda_{\overline{0}}\right) \Upsilon_{0}\left(\lambda_{1}, \lambda_{\overline{0}}\right)}{\mathcal{W}\left(\lambda_{1}, \lambda_{\overline{0}}\right)} \mathcal{H}\left(\mathrm{X}_{1} \mid \lambda_{1}, \lambda_{\overline{0}}\right),
\end{aligned}
$$

with

$$
\begin{aligned}
\mathcal{W}\left(\lambda_{0}, \lambda_{1}\right):= & \operatorname{det}\left(\begin{array}{ll}
\Omega_{0}\left(\lambda_{0}, \lambda_{1}\right) & \Omega_{1}\left(\lambda_{0}, \lambda_{1}\right) \\
\Omega_{0}\left(\lambda_{0}, \lambda_{1}\right) & \bar{\Omega}_{1}\left(\lambda_{0}, \lambda_{1}\right)
\end{array}\right) \\
= & \frac{\left[1-q^{2} e^{2\left(\lambda_{0}-\lambda_{1}\right)}\right]^{2}\left[1-\zeta e^{2\left(\lambda_{0}-\lambda_{1}\right)}\right]^{2}}{\left[e^{2\left(\lambda_{0}-\lambda_{1}\right)}-1\right]^{2}\left[q^{2}-\zeta e^{2\left(\lambda_{0}-\lambda_{1}\right)}\right]} \frac{\Lambda\left(\lambda_{0}\right) \bar{\Lambda}\left(\lambda_{1}\right)}{q^{\frac{1}{2}}\left(q^{2}-1\right)} \\
& -\frac{\left[1-\zeta e^{2\left(\lambda_{0}-\lambda_{1}\right)}\right]^{2}\left[q^{4}-\zeta^{2} e^{2\left(\lambda_{0}-\lambda_{1}\right)}\right]^{2}}{\zeta^{2}\left[e^{2\left(\lambda_{0}-\lambda_{1}\right)}-1\right]^{2}\left[q^{2}-\zeta e^{2\left(\lambda_{0}-\lambda_{1}\right)}\right]} \frac{\Lambda\left(\lambda_{1}\right) \bar{\Lambda}\left(\lambda_{0}\right)}{q^{\frac{5}{2}}\left(q^{2}-1\right)} .
\end{aligned}
$$

As for the aforementioned relation between $\mathcal{H}$ and $\overline{\mathcal{H}}$, it is readily obtained through the identity $\mathcal{Z}(\mathrm{X})=\mathcal{Z}(\mathrm{X})$ using $(4.8)$ and (4.9). In fact, different relations between $\mathcal{H}$ and $\overline{\mathcal{H}}$ could also be obtained from (4.8) and (4.9). For instance, one finds a differential equation through the obvious identity $\partial \mathcal{Z}(\mathrm{X}) / \partial \lambda_{0}=0$ with $\mathcal{Z}(\mathrm{X})$ given by (4.8) and also $\partial \mathcal{Z}(\mathrm{X}) / \partial \lambda_{\overline{0}}=0$ using (4.9). Moreover, from (4.8) and/or (4.9), one can clearly see that the partition function $\mathcal{Z}$ is fixed once we determine the functions $\mathcal{H}$ and $\mathcal{H}$. Consequently, we shall then discuss the explicit resolution of the $\mathrm{ZH}$-system for lattice lengths $L=1,2,3$.

\section{B. Case $L=1$}

This is the simplest instance of the $\mathrm{ZH}$-system, and its analysis requires only trivial considerations given the results already obtained in Secs. II, III, and IV. For instance, Eq. (4.7) for $L=1$ gives

$$
\omega\left(\lambda_{1}\right) \mathcal{H}\left(\emptyset \mid \lambda_{1}, \lambda_{0}\right)=\bar{\omega}\left(\lambda_{0}\right) \overline{\mathcal{H}}\left(\lambda_{1}, \lambda_{0} \mid \emptyset\right),
$$

which corresponds to the condition $\mathcal{F}=\overline{\mathcal{F}}$ expected from formulas (2.19). Now, taking into account the polynomial structure described in Corollaries 2.7 and 2.9 , identity (4.11) allows us to conclude

$$
\mathcal{H}\left(\emptyset \mid \lambda_{1}, \lambda_{0}\right)=\kappa \bar{\omega}\left(\lambda_{0}\right) \quad \text { and } \quad \overline{\mathcal{H}}\left(\lambda_{1}, \lambda_{0} \mid \emptyset\right)=\kappa \omega\left(\lambda_{1}\right),
$$

with $\kappa \in \mathbb{C}$ being a constant yet to be determined. We can then simply substitute formulas (4.12) in (4.8) or (4.9) to find the partition function $\mathcal{Z}$ up to the overall multiplicative factor $\kappa$. The latter is then fixed by the initial condition (2.33), and we end up with $\mathcal{Z}\left(\lambda_{1}\right)=d_{1,3}\left(\lambda_{1}-\mu_{1}\right)$ as expected.

\section{Case $L=\mathbf{2}$}

Our goal in explicitly solving the ZH-system for small values of the lattice length $L$ is to provide evidences that our system of equations indeed constrains the partition functions $\mathcal{Z}, \mathcal{H}$, and $\overline{\mathcal{H}}$ up to an overall multiplicative constant. In particular, we are interested in showing the existence of unique polynomial solutions with structure described in Lemmas 2.3 and 2.5. Therefore, taking into account the aforementioned lemmas as well as Corollaries 2.7 and 2.9 , we can write for $L=2$,

$$
\begin{aligned}
& \mathcal{H}\left(\lambda_{0} \mid \lambda_{1}, \lambda_{2}\right)=\sum_{i=0}^{3} \sum_{j=0}^{1} \sum_{k=0}^{3} \phi_{i, j, k} e^{2 i \lambda_{0}+2 j \lambda_{1}+2 k \lambda_{2}}, \\
& \overline{\mathcal{H}}\left(\lambda_{0}, \lambda_{1} \mid \lambda_{2}\right)=\sum_{i=0}^{3} \sum_{j=0}^{1} \sum_{k=0}^{3} \bar{\phi}_{i, j, k} e^{2 i \lambda_{0}+2 j \lambda_{1}+2 k \lambda_{2}},
\end{aligned}
$$

with coefficients $\phi_{i, j, k}$ and $\bar{\phi}_{i, j, k}$ still being undetermined. Although the partition function $\mathcal{Z}$ is also a polynomial according to Lemma 2.3, one can directly read it off from formulas (4.8) and (4.9). In this way, we can restrict our attention to the functions $\mathcal{H}$ and $\overline{\mathcal{H}}$ in order to solve the ZH-system. 
Now turning our attention to the $\mathrm{ZH}$-system, it is then convenient to eliminate the function $\mathcal{Z}$ from our problem. The latter task can then be simply accomplished through the identification of (4.8) and (4.9). Therefore, we are left with a reduced system of equations reading

$$
\begin{gathered}
\quad \frac{\Upsilon_{0}\left(\lambda_{0}, \lambda_{1}\right) \bar{\Omega}_{1}\left(\lambda_{0}, \lambda_{1}\right)}{\mathcal{W}\left(\lambda_{0}, \lambda_{1}\right)} \mathcal{H}\left(\lambda_{2} \mid \lambda_{0}, \lambda_{1}\right)+\frac{\Upsilon_{1}\left(\lambda_{0}, \lambda_{1}\right) \bar{\Omega}_{1}\left(\lambda_{0}, \lambda_{1}\right)}{\mathcal{W}\left(\lambda_{0}, \lambda_{1}\right)} \mathcal{H}\left(\lambda_{2} \mid \lambda_{1}, \lambda_{0}\right) \\
-\frac{\bar{\Upsilon}_{0}\left(\lambda_{0}, \lambda_{1}\right) \Omega_{1}\left(\lambda_{0}, \lambda_{1}\right)}{\mathcal{W}\left(\lambda_{0}, \lambda_{1}\right)} \overline{\mathcal{H}}\left(\lambda_{1}, \lambda_{0} \mid \lambda_{2}\right)-\frac{\bar{\Upsilon}_{1}\left(\lambda_{0}, \lambda_{1}\right) \Omega_{1}\left(\lambda_{0}, \lambda_{1}\right)}{\mathcal{W}\left(\lambda_{0}, \lambda_{1}\right)} \overline{\mathcal{H}}\left(\lambda_{0}, \lambda_{1} \mid \lambda_{2}\right)= \\
\frac{\Omega_{0}\left(\lambda_{1}, \lambda_{\overline{0}}\right) \bar{\Upsilon}_{1}\left(\lambda_{1}, \lambda_{\overline{0}}\right)}{\mathcal{W}\left(\lambda_{1}, \lambda_{\overline{0}}\right)} \overline{\mathcal{H}}\left(\lambda_{1}, \lambda_{\overline{0}} \mid \lambda_{2}\right)+\frac{\Omega_{0}\left(\lambda_{1}, \lambda_{\overline{0}}\right) \bar{\Upsilon}_{0}\left(\lambda_{1}, \lambda_{\overline{0}}\right)}{\mathcal{W}\left(\lambda_{1}, \lambda_{\overline{0}}\right)} \overline{\mathcal{H}}\left(\lambda_{\overline{0}}, \lambda_{1} \mid \lambda_{2}\right) \\
-\frac{\bar{\Omega}_{0}\left(\lambda_{1}, \lambda_{\overline{0}}\right) \Upsilon_{1}\left(\lambda_{1}, \lambda_{\overline{0}}\right)}{\mathcal{W}\left(\lambda_{1}, \lambda_{\overline{0}}\right)} \mathcal{H}\left(\lambda_{2} \mid \lambda_{\overline{0}}, \lambda_{1}\right)-\frac{\bar{\Omega}_{0}\left(\lambda_{1}, \lambda_{\overline{0}}\right) \Upsilon_{0}\left(\lambda_{1}, \lambda_{\overline{0}}\right)}{\mathcal{W}\left(\lambda_{1}, \lambda_{\overline{0}}\right)} \mathcal{H}\left(\lambda_{2} \mid \lambda_{1}, \lambda_{\overline{0}}\right), \\
\bar{\omega}\left(\lambda_{2}\right) \overline{\mathcal{H}}\left(\lambda_{0}, \lambda_{2} \mid \lambda_{1}\right)=\mathcal{M}_{0}^{(1)} \omega\left(\lambda_{0}\right)\left[\mathcal{N}_{0,1}^{(1)} \mathcal{H}\left(\lambda_{2} \mid \lambda_{0}, \lambda_{1}\right)+\mathcal{N}_{0,2}^{(1)} \mathcal{H}\left(\lambda_{1} \mid \lambda_{0}, \lambda_{2}\right)\right] \\
+\mathcal{M}_{1}^{(1)} \omega\left(\lambda_{1}\right)\left[\mathcal{N}_{1,0}^{(1)} \mathcal{H}\left(\lambda_{2} \mid \lambda_{1}, \lambda_{0}\right)+\mathcal{N}_{1,2}^{(1)} \mathcal{H}\left(\lambda_{0} \mid \lambda_{1}, \lambda_{2}\right)\right],
\end{gathered}
$$

for $L=2$. The latter then involves only the functions $\mathcal{H}$ and $\overline{\mathcal{H}}$.

The system of equations (4.9) is linear, and the use of expressions (4.13) will consequently yield linear algebraic equations for the coefficients $\phi_{i, j, k}$ and $\bar{\phi}_{i, j, k}$. In this way, the existence of unique trigonometric polynomials $\mathcal{H}$ and $\mathcal{H}$ (up to an overall constant) solving (4.14) will depend on having enough independent equations constraining our coefficients. Moreover, it is important to emphasize that (4.14) is a system of equations on the variables $\lambda_{0}, \lambda_{1}$, and $\lambda_{2}$ although the coefficients in (4.14) also depend on the inhomogeneity parameters $\mu_{1}$ and $\mu_{2}$. In this way, by setting $\mu_{i}$ to particular values, we could only decrease the number of linearly independent equations while keeping the same number of coefficients $\phi_{i, j, k}$ and $\bar{\phi}_{i, j, k}$. Therefore, if we already find enough constraints for particular values of the anisotropy parameter, then it is certainly enough for generic values of the latter since we would have at least the same number of equations. We then proceed by fixing $\mu_{i}=0$ for simplicity reasons and present the coefficients $\phi_{i, j, k}$ and $\bar{\phi}_{i, j, k}$ obtained from the resolution of (4.9) in Appendix B.

\section{Case $L=3$}

As for the case $L=3$, we proceed along the same lines employed in Sec. IV C for $L=2$. We then start by considering a reduced version of the $\mathrm{ZH}$-system obtained through the elimination of the partition function $\mathcal{Z}$. More precisely, we consider the equation resulting from the identification of (4.8) and (4.9), in addition to the second relation in (4.7) for $L=3$. The latter equations read

$$
\begin{array}{r}
\quad \frac{\Upsilon_{0}\left(\lambda_{0}, \lambda_{1}\right) \bar{\Omega}_{1}\left(\lambda_{0}, \lambda_{1}\right)}{\mathcal{W}\left(\lambda_{0}, \lambda_{1}\right)} \mathcal{H}\left(\lambda_{2}, \lambda_{3} \mid \lambda_{0}, \lambda_{1}\right)+\frac{\Upsilon_{1}\left(\lambda_{0}, \lambda_{1}\right) \bar{\Omega}_{1}\left(\lambda_{0}, \lambda_{1}\right)}{\mathcal{W}\left(\lambda_{0}, \lambda_{1}\right)} \mathcal{H}\left(\lambda_{2}, \lambda_{3} \mid \lambda_{1}, \lambda_{0}\right) \\
-\frac{\bar{\Upsilon}_{0}\left(\lambda_{0}, \lambda_{1}\right) \Omega_{1}\left(\lambda_{0}, \lambda_{1}\right)}{\mathcal{W}\left(\lambda_{0}, \lambda_{1}\right)} \overline{\mathcal{H}}\left(\lambda_{1}, \lambda_{0} \mid \lambda_{2}, \lambda_{3}\right)-\frac{\bar{\Upsilon}_{1}\left(\lambda_{0}, \lambda_{1}\right) \Omega_{1}\left(\lambda_{0}, \lambda_{1}\right)}{\mathcal{W}\left(\lambda_{0}, \lambda_{1}\right)} \overline{\mathcal{H}}\left(\lambda_{0}, \lambda_{1} \mid \lambda_{2}, \lambda_{3}\right)= \\
\quad \frac{\Omega_{0}\left(\lambda_{1}, \lambda_{\overline{0}}\right) \bar{\Upsilon}_{1}\left(\lambda_{1}, \lambda_{\overline{0}}\right)}{\mathcal{W}\left(\lambda_{1}, \lambda_{\overline{0}}\right)} \overline{\mathcal{H}}\left(\lambda_{1}, \lambda_{\overline{0}} \mid \lambda_{2}, \lambda_{3}\right)+\frac{\Omega_{0}\left(\lambda_{1}, \lambda_{\overline{0}}\right) \bar{\Upsilon}_{0}\left(\lambda_{1}, \lambda_{\overline{0}}\right)}{\mathcal{W}\left(\lambda_{1}, \lambda_{\overline{0}}\right)} \overline{\mathcal{H}}\left(\lambda_{\overline{0}}, \lambda_{1} \mid \lambda_{2}, \lambda_{3}\right) \\
-\frac{\bar{\Omega}_{0}\left(\lambda_{1}, \lambda_{\overline{0}}\right) \Upsilon_{1}\left(\lambda_{1}, \lambda_{\overline{0}}\right)}{\mathcal{W}\left(\lambda_{1}, \lambda_{\overline{0}}\right)} \mathcal{H}\left(\lambda_{2}, \lambda_{3} \mid \lambda_{\overline{0}}, \lambda_{1}\right)-\frac{\bar{\Omega}_{0}\left(\lambda_{1}, \lambda_{\overline{0}}\right) \Upsilon_{0}\left(\lambda_{1}, \lambda_{\overline{0}}\right)}{\mathcal{W}\left(\lambda_{1}, \lambda_{\overline{0}}\right)} \mathcal{H}\left(\lambda_{2}, \lambda_{3} \mid \lambda_{1}, \lambda_{\overline{0}}\right) \\
\bar{\omega}\left(\lambda_{3}\right) \overline{\mathcal{H}}\left(\lambda_{0}, \lambda_{3} \mid \lambda_{1}, \lambda_{2}\right)= \\
\mathcal{M}_{0}^{(2)} \omega\left(\lambda_{0}\right)\left[\mathcal{N}_{0,1}^{(2)} \mathcal{H}\left(\lambda_{2}, \lambda_{3} \mid \lambda_{0}, \lambda_{1}\right)+\mathcal{N}_{0,2}^{(2)} \mathcal{H}\left(\lambda_{1}, \lambda_{3} \mid \lambda_{0}, \lambda_{2}\right)+\mathcal{N}_{0,3}^{(2)} \mathcal{H}\left(\lambda_{1}, \lambda_{2} \mid \lambda_{0}, \lambda_{3}\right)\right] \\
+\mathcal{M}_{1}^{(2)} \omega\left(\lambda_{1}\right)\left[\mathcal{N}_{1,0}^{(2)} \mathcal{H}\left(\lambda_{2}, \lambda_{3} \mid \lambda_{1}, \lambda_{0}\right)+\mathcal{N}_{1,2}^{(2)} \mathcal{H}\left(\lambda_{0}, \lambda_{3} \mid \lambda_{1}, \lambda_{2}\right)+\mathcal{N}_{1,3}^{(2)} \mathcal{H}\left(\lambda_{0}, \lambda_{2} \mid \lambda_{1}, \lambda_{3}\right)\right] \\
+\mathcal{M}_{2}^{(2)} \omega\left(\lambda_{2}\right)\left[\mathcal{N}_{2,0}^{(2)} \mathcal{H}\left(\lambda_{1}, \lambda_{3} \mid \lambda_{2}, \lambda_{0}\right)+\mathcal{N}_{2,1}^{(2)} \mathcal{H}\left(\lambda_{0}, \lambda_{3} \mid \lambda_{2}, \lambda_{1}\right)+\mathcal{N}_{2,3}^{(2)} \mathcal{H}\left(\lambda_{0}, \lambda_{1} \mid \lambda_{2}, \lambda_{3}\right)\right],
\end{array}
$$

and it is worth remarking that the function $\mathcal{H}$ is symmetric on the first two arguments, while $\overline{\mathcal{H}}$ is symmetric on the last two arguments.

Next, we write

$$
\begin{aligned}
& \mathcal{H}\left(\lambda_{0}, \lambda_{1} \mid \lambda_{2}, \lambda_{3}\right)=\sum_{i=0}^{5} \sum_{j=0}^{5} \sum_{k=0}^{2} \sum_{l=0}^{5} \phi_{i, j, k, l} e^{2 i \lambda_{0}+2 j \lambda_{1}+2 k \lambda_{2}+2 l \lambda_{3}}, \\
& \overline{\mathcal{H}}\left(\lambda_{0}, \lambda_{1} \mid \lambda_{2}, \lambda_{3}\right)=\sum_{i=0}^{5} \sum_{j=0}^{2} \sum_{k=0}^{5} \sum_{l=0}^{5} \bar{\phi}_{i, j, k, l} e^{2 i \lambda_{0}+2 j \lambda_{1}+2 k \lambda_{2}+2 l \lambda_{3}},
\end{aligned}
$$


in accordance with the polynomial structure discussed in Sec. II E. Moreover, for our purposes here, we can also set $\mu_{j}=0$ using the same arguments discussed in Sec. IV C for the case $L=2$. In this way, the substitution of (4.16) into the system of functional equations (4.15) yields a system of linear algebraic equations for the coefficients $\phi_{i, j, k, l}$ and $\bar{\phi}_{i, j, k, l}$. The resolution of the latter shows that all coefficients $\phi_{i, j, k, l}$ and $\bar{\phi}_{i, j, k, l}$ are fixed except for one. Due to the large amount of coefficients present in this case, we have preferred not to display the solutions as we did for the case $L=2$. However, our results for the case $L=3$ also corroborate our claim that the $\mathrm{ZH}$-system is sufficient to characterize the partition function $\mathcal{Z}$ up to an overall multiplicative factor.

\section{CONCLUDING REMARKS}

The main result of this paper is the system of functional equations formed by (4.3), (4.5), and (4.7) describing partition functions of two integrable nineteen-vertex models, namely, the IK and FZ models, with three different types of domain-wall boundary conditions. We refer to such a system of equations as the $\mathrm{ZH}$-system, and it provides a relation between the partition functions $\mathcal{Z}, \mathcal{F}$, and $\overline{\mathcal{F}}$ defined in $(2.19)$ in an algebraic manner. A schematic representation of the $\mathrm{ZH}$-system is shown in Fig. 10.

The Yang-Baxter algebra attached to nineteen-vertex models is the origin of the ZH-system, and the derivation of the latter system of equations follows the AF method previously devised for six-vertex models. Although the idea employed here is essentially the same as the one used in the case of the six-vertex model, the derivation of the $\mathrm{ZH}$-system still encompasses some additional mechanisms. For instance, one can notice the similar role played by the functions $\Lambda(\bar{\Lambda})$ and $\omega(\bar{\omega})$ defined in (4.2) in the coefficients of (4.3) and (4.5). In the case of the six-vertex model, we only have the presence of terms with the same origin as $\Lambda$ and $\bar{\Lambda}$, which are direct consequences of the weight-modules discussed in Sec. III C. The terms $\omega$ and $\bar{\omega}$, in their turn, arise from the existence of simple zeroes as shown by Lemmas 2.6 and 2.8.

At first look, the structure of the $\mathrm{ZH}$-system seems to be completely different from the functional equations obtained for the six-vertex model. However, there are still important similarities worth remarking. For instance, the relations between the functions $\mathcal{H}$ and $\overline{\mathcal{H}}$ stated in Lemma 4.3 can be regarded as a doubled and inhomogeneous version of the six-vertex model's equations. This is essentially due to the commutation relations (3.8), which can be recognized as the same relations appearing in the six-vertex model.

Although the partition functions $\mathcal{Z}, \mathcal{F}$, and $\overline{\mathcal{F}}$ satisfy the $\mathrm{ZH}$-system by construction, it is not a priori clear if our system of equations is indeed capable of fixing the aforementioned quantities uniquely. Therefore, in Secs. IV B-IV D, we have discussed the explicit resolution of the ZH-system for lattice lengths $L=1,2,3$, taking into account the polynomial structure expected from our partition functions. This explicit analysis for small lattice lengths shows that the $\mathrm{ZH}$-system is indeed capable of fixing our partition functions up to an overall multiplicative constant. The latter can then be fixed by evaluating any of our three partition functions at particular values of the spectral parameters. In Sec. II $\mathrm{G}$, we have then found that the specialization $\mathcal{Z}\left(\mu_{1}, \mu_{2}, \ldots, \mu_{L}\right)$ can easily be obtained and used (2.33) to fix the overall constant. The latter overall factor is not a fundamental quantity from the statistical mechanics point of view, but it becomes relevant, for instance, for possible applications in enumerative combinatorics along the lines of Ref. 41.

Moreover, the explicit inspection of the ZH-system for $L=2$ presented in Sec. IV C and Appendix B shows an interesting difference between the IK and FZ models considered in this work. For instance, one can notice that several coefficients of the functions $\mathcal{H}$ and $\overline{\mathcal{H}}$ vanish for the FZ model, while they are all different from zero for the IK model. Such functions are the building blocks of the partition function $\mathcal{Z}$, as it can be seen from formulas (4.8) and (4.9), and this feature might justify the possibility of expressing $\mathcal{Z}$ for the FZ model as a determinant according to Ref. 31. As far as the general solution of the ZH-system is concerned, this problem has eluded us so far but we hope the methods put forward in Refs. 29, 30, 27, and 35 shed some light onto possible multiple contour integral or determinantal solutions.

\section{ACKNOWLEDGMENTS}

The authors thank N. Beisert for discussions and comments. The work of W.G. is partially supported by the Swiss National Science Foundation through the NCCR SwissMAP and by Grant No. 615203 from the European Research Council under the FP7.

\section{APPENDIX A: THE SUBALGEBRA $\mathscr{S}_{\mathcal{A}, \mathcal{B}, \mathcal{E}}$}

As for nineteen-vertex models described by the $\mathcal{R}$-matrix (2.11), the associated Yang-Baxter algebra comprises 81 commutation relations involving the entries of the monodromy matrix (2.8). Here, however, we are interested only in the subalgebra spanned by the generators $\mathcal{A}$,

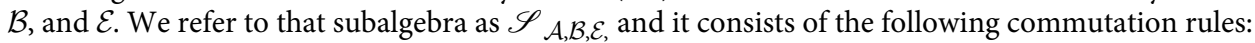

$$
\begin{aligned}
\mathcal{A}\left(\lambda_{1}\right) \mathcal{A}\left(\lambda_{2}\right) & =\mathcal{A}\left(\lambda_{2}\right) \mathcal{A}\left(\lambda_{1}\right) \\
\mathcal{A}\left(\lambda_{1}\right) \mathcal{B}\left(\lambda_{2}\right) & =\frac{a\left(\lambda_{2}-\lambda_{1}\right)}{b\left(\lambda_{2}-\lambda_{1}\right)} \mathcal{B}\left(\lambda_{2}\right) \mathcal{A}\left(\lambda_{1}\right)-\frac{c\left(\lambda_{2}-\lambda_{1}\right)}{b\left(\lambda_{2}-\lambda_{1}\right)} \mathcal{B}\left(\lambda_{1}\right) \mathcal{A}\left(\lambda_{2}\right) \\
\mathcal{A}\left(\lambda_{1}\right) \mathcal{E}\left(\lambda_{2}\right)= & \frac{a\left(\lambda_{2}-\lambda_{1}\right)}{d_{3,3}\left(\lambda_{2}-\lambda_{1}\right)} \mathcal{E}\left(\lambda_{2}\right) \mathcal{A}\left(\lambda_{1}\right)-\frac{d_{1,3}\left(\lambda_{2}-\lambda_{1}\right)}{d_{3,3}\left(\lambda_{2}-\lambda_{1}\right)} \mathcal{E}\left(\lambda_{1}\right) \mathcal{A}\left(\lambda_{2}\right) \\
& -\frac{d_{2,3}\left(\lambda_{2}-\lambda_{1}\right)}{d_{3,3}\left(\lambda_{2}-\lambda_{1}\right)} \mathcal{B}\left(\lambda_{1}\right) \mathcal{B}\left(\lambda_{2}\right)
\end{aligned}
$$




$$
\begin{aligned}
& \mathcal{B}\left(\lambda_{1}\right) \mathcal{A}\left(\lambda_{2}\right)= \frac{a\left(\lambda_{2}-\lambda_{1}\right)}{b\left(\lambda_{2}-\lambda_{1}\right)} \mathcal{A}\left(\lambda_{2}\right) \mathcal{B}\left(\lambda_{1}\right)-\frac{\bar{c}\left(\lambda_{2}-\lambda_{1}\right)}{b\left(\lambda_{2}-\lambda_{1}\right)} \mathcal{A}\left(\lambda_{1}\right) \mathcal{B}\left(\lambda_{2}\right) \\
& \mathcal{B}\left(\lambda_{1}\right) \mathcal{B}\left(\lambda_{2}\right)= \frac{a\left(\lambda_{2}-\lambda_{1}\right)}{d_{2,1}\left(\lambda_{2}-\lambda_{1}\right)} \mathcal{A}\left(\lambda_{2}\right) \mathcal{E}\left(\lambda_{1}\right)-\frac{d_{3,1}\left(\lambda_{2}-\lambda_{1}\right)}{d_{2,1}\left(\lambda_{2}-\lambda_{1}\right)} \mathcal{A}\left(\lambda_{1}\right) \mathcal{E}\left(\lambda_{2}\right) \\
&-\frac{d_{1,1}\left(\lambda_{2}-\lambda_{1}\right)}{d_{2,1}\left(\lambda_{2}-\lambda_{1}\right)} \mathcal{E}\left(\lambda_{1}\right) \mathcal{A}\left(\lambda_{2}\right) \\
& \mathcal{B}\left(\lambda_{1}\right) \mathcal{E}\left(\lambda_{2}\right)= \frac{a\left(\lambda_{2}-\lambda_{1}\right)}{b\left(\lambda_{2}-\lambda_{1}\right)} \mathcal{E}\left(\lambda_{2}\right) \mathcal{B}\left(\lambda_{1}\right)-\frac{c\left(\lambda_{2}-\lambda_{1}\right)}{b\left(\lambda_{2}-\lambda_{1}\right)} \mathcal{E}\left(\lambda_{1}\right) \mathcal{B}\left(\lambda_{2}\right) \\
& \mathcal{E}\left(\lambda_{1}\right) \mathcal{A}\left(\lambda_{2}\right)=\frac{a\left(\lambda_{2}-\lambda_{1}\right)}{d_{1,2}\left(\lambda_{2}-\lambda_{1}\right)} \mathcal{B}\left(\lambda_{2}\right) \mathcal{B}\left(\lambda_{1}\right)-\frac{d_{2,2}\left(\lambda_{2}-\lambda_{1}\right)}{d_{1,2}\left(\lambda_{2}-\lambda_{1}\right)} \mathcal{B}\left(\lambda_{1}\right) \mathcal{B}\left(\lambda_{2}\right) \\
& \quad-\frac{d_{3,2}\left(\lambda_{2}-\lambda_{1}\right)}{d_{1,2}\left(\lambda_{2}-\lambda_{1}\right)} \mathcal{A}\left(\lambda_{1}\right) \mathcal{E}\left(\lambda_{2}\right) \\
& \mathcal{E}\left(\lambda_{1}\right) \mathcal{B}\left(\lambda_{2}\right)=\frac{a\left(\lambda_{2}-\lambda_{1}\right)}{b\left(\lambda_{2}-\lambda_{1}\right)} \mathcal{B}\left(\lambda_{2}\right) \mathcal{E}\left(\lambda_{1}\right)-\frac{\bar{c}\left(\lambda_{2}-\lambda_{1}\right)}{b\left(\lambda_{2}-\lambda_{1}\right)} \mathcal{B}\left(\lambda_{1}\right) \mathcal{E}\left(\lambda_{2}\right) \\
& \mathcal{E}\left(\lambda_{1}\right) \mathcal{E}\left(\lambda_{2}\right)= \mathcal{E}\left(\lambda_{2}\right) \mathcal{E}\left(\lambda_{1}\right) .
\end{aligned}
$$

\begin{tabular}{|c|c|c|c|c|c|c|c|}
\hline $\mathrm{i}$ & j & $\mathrm{k}$ & $\phi_{i, j, k} / \phi_{0,0,0}$ & $\mathrm{i}$ & j & $\mathrm{k}$ & $\phi_{i, j, k} / \phi_{0,0,0}$ \\
\hline 0 & 0 & 0 & 1 & 0 & 1 & 0 & $-\frac{2}{q^{2}+1}$ \\
\hline 0 & 0 & 1 & $-\frac{2\left(2 q^{4}-2 q-1\right)}{q^{2}\left(q^{2}+1\right)\left(q^{2}+q+1\right)}$ & 0 & 1 & 1 & $\frac{q^{7}+q^{6}+5 q^{5}-3 q^{4}-4 q^{3}-3 q^{2}+1}{q^{5}\left(q^{2}+1\right)\left(q^{2}+q+1\right)}$ \\
\hline 0 & 0 & 2 & $\frac{q^{7}+q^{5}-8 q^{4}-3 q^{3}+q^{2}+q+1}{q^{5}\left(q^{2}+1\right)\left(q^{2}+q+1\right)}$ & 0 & 1 & 2 & $-\frac{2\left(q^{5}-q^{4}-q^{3}-4 q^{2}+2\right)}{q^{5}\left(q^{2}+1\right)\left(q^{2}+q+1\right)}$ \\
\hline 0 & 0 & 3 & $\frac{2\left(q^{4}-q-1\right)}{q^{5}\left(q^{2}+1\right)\left(q^{2}+q+1\right)}$ & 0 & 1 & 3 & $-\frac{3 q^{4}-q^{3}-2 q^{2}-q-1}{q^{7}\left(q^{2}+1\right)\left(q^{2}+q+1\right)}$ \\
\hline 1 & 0 & 0 & $-\frac{2\left(q^{4}-2 q^{3}-2 q+1\right)}{(q-1) q\left(q^{2}+1\right)\left(q^{2}+q+1\right)}$ & 1 & 1 & 0 & $\frac{q^{6}+q^{4}-8 q^{3}+q^{2}+1}{q^{3}\left(q^{5}+q^{3}-q^{2}-1\right)}$ \\
\hline 1 & 0 & 1 & $-\frac{q^{10}-4 q^{8}+5 q^{7}-q^{6}+17 q^{5}-13 q^{4}-3 q^{3}+q+1}{q^{5}\left(q^{5}+q^{3}-q^{2}-1\right)}$ & 1 & 1 & 1 & $-\frac{2\left(2 q^{6}-7 q^{5}+q^{4}-4 q^{3}+8 q^{2}+q-3\right)}{q^{5}\left(q^{5}+q^{3}-q^{2}-1\right)}$ \\
\hline 1 & 0 & 2 & $-\frac{2\left(q^{7}-8 q^{6}+7 q^{5}+q^{4}+5 q^{3}-2 q^{2}-3 q+1\right)}{q^{6}\left(q^{5}+q^{3}-q^{2}-1\right)}$ & 1 & 1 & 2 & $\frac{q^{11}-5 q^{10}+2 q^{9}-12 q^{8}+22 q^{7}+q^{6}-3 q^{4}-5 q^{3}+4 q^{2}-1}{q^{10}\left(q^{5}+q^{3}-q^{2}-1\right)}$ \\
\hline 1 & 0 & 3 & $-\frac{q^{8}-4 q^{7}+2 q^{6}-8 q^{5}+2 q^{4}+4 q^{3}-1}{q^{8}\left(q^{5}+q^{3}-q^{2}-1\right)}$ & 1 & 1 & 3 & $\frac{2\left(q^{6}-4 q^{5}+q^{4}-2 q^{3}+q^{2}+2 q-1\right)}{q^{8}\left(q^{5}+q^{3}-q^{2}-1\right)}$ \\
\hline 2 & 0 & 0 & $-\frac{q^{4}+q^{3}+6 q^{2}+q+1}{q^{3}\left(q^{2}+1\right)\left(q^{2}+q+1\right)}$ & 2 & 1 & 0 & $\frac{2\left(2 q^{2}+q+2\right)}{q^{3}\left(q^{2}+1\right)\left(q^{2}+q+1\right)}$ \\
\hline 2 & 0 & 1 & $\frac{2\left(6 q^{4}-q^{3}-3 q-1\right)}{q^{5}\left(q^{2}+1\right)\left(q^{2}+q+1\right)}$ & 2 & 1 & 1 & $-\frac{5 q^{7}+q^{6}+9 q^{5}-7 q^{4}-4 q^{3}-3 q^{2}+1}{q^{8}\left(q^{2}+1\right)\left(q^{2}+q+1\right)}$ \\
\hline 2 & 0 & 2 & $-\frac{5 q^{7}-4 q^{6}+q^{5}-12 q^{4}-3 q^{3}+q^{2}+q+1}{q^{8}\left(q^{2}+1\right)\left(q^{2}+q+1\right)}$ & 2 & 1 & 2 & $\frac{2\left(q^{7}+2 q^{5}-5 q^{4}-q^{3}-4 q^{2}+2\right)}{q^{8}\left(q^{2}+1\right)\left(q^{2}+q+1\right)}$ \\
\hline 2 & 0 & 3 & $-\frac{2\left(q^{6}+2 q^{4}-q-1\right)}{q^{8}\left(q^{2}+1\right)\left(q^{2}+q+1\right)}$ & 2 & 1 & 3 & $\frac{4 q^{4}-q^{2}-q-1}{q^{10}\left(q^{2}+q+1\right)}$ \\
\hline 3 & 0 & 0 & $\frac{2}{q^{3}-q^{6}}$ & 3 & 1 & 0 & $\frac{q^{2}+1}{q^{5}\left(q^{3}-1\right)}$ \\
\hline 3 & 0 & 1 & $\frac{4 q^{3}-q^{2}-1}{q^{6}\left(q^{3}-1\right)}$ & 3 & 1 & 1 & $-\frac{2\left(q^{3}+q-1\right)}{q^{6}\left(q^{3}-1\right)}$ \\
\hline 3 & 0 & 2 & $-\frac{2\left(q^{3}-q^{2}-1\right)}{q^{6}\left(q^{3}-1\right)}$ & 3 & 1 & 2 & $\frac{q^{3}+q-4}{q^{6}\left(q^{3}-1\right)}$ \\
\hline 3 & 0 & 3 & $-\frac{q^{2}+1}{q^{6}\left(q^{3}-1\right)}$ & 3 & 1 & 3 & $\frac{2}{q^{6}\left(q^{3}-1\right)}$ \\
\hline
\end{tabular}

\section{APPENDIX B: COEFFICIENTS $\phi_{i, j, k}$ AND $\bar{\phi}_{i j, k}$}

In this appendix, we present the coefficients for the functions $\mathcal{H}$ and $\overline{\mathcal{H}}$ according to formulas (4.13) obtained through the resolution of the ZH-system for the case $L=2$. In particular, we explicit the coefficients $\phi_{i, j, k}$ and $\bar{\phi}_{i, j, k}$ for the IK model in Tables I and II, respectively. Tables III and IV then contain, respectively, the results for $\phi_{i, j, k}$ and $\bar{\phi}_{i, j, k}$ associated with the FZ model.

TABLE I. Coefficients of the function $\mathcal{H}$ for the IK model with $L=2$. 
TABLE II. Coefficients of the function $\overline{\mathcal{H}}$ for the IK model with $L=2$.

\begin{tabular}{|c|c|c|c|c|c|c|c|}
\hline $\mathrm{i}$ & $\mathrm{j}$ & $\mathrm{k}$ & $\bar{\phi}_{i, j, k} / \phi_{0,0,0}$ & $\mathrm{i}$ & j & $\mathrm{k}$ & $\bar{\phi}_{i, j, k} / \phi_{0,0,0}$ \\
\hline 0 & 0 & 0 & $q^{4}$ & 0 & 1 & 0 & $\frac{2 q^{3}}{q^{2}+1}$ \\
\hline 0 & 0 & 1 & $-\frac{2 q^{3}\left(q^{4}-2 q^{3}-2 q+1\right)}{(q-1)\left(q^{2}+1\right)\left(q^{2}+q+1\right)}$ & 0 & 1 & 1 & $-\frac{q^{6}+q^{4}-8 q^{3}+q^{2}+1}{(q-1)\left(q^{2}+1\right)\left(q^{2}+q+1\right)}$ \\
\hline 0 & 0 & 2 & $-\frac{q\left(q^{4}+q^{3}+6 q^{2}+q+1\right)}{\left(q^{2}+1\right)\left(q^{2}+q+1\right)}$ & 0 & 1 & 2 & $-\frac{2\left(2 q^{2}+q+2\right)}{\left(q^{2}+1\right)\left(q^{2}+q+1\right)}$ \\
\hline 0 & 0 & 3 & $-\frac{2 q}{(q-1)\left(q^{2}+q+1\right)}$ & 0 & 1 & 3 & $-\frac{q^{2}+1}{(q-1) q^{2}\left(q^{2}+q+1\right)}$ \\
\hline 1 & 0 & 0 & $-\frac{2 q^{3}\left(q^{4}+2 q^{3}-2\right)}{\left(q^{2}+1\right)\left(q^{2}+q+1\right)}$ & 1 & 1 & 0 & $\frac{q^{7}-3 q^{5}-4 q^{4}-3 q^{3}+5 q^{2}+q+1}{\left(q^{2}+1\right)\left(q^{2}+q+1\right)}$ \\
\hline 1 & 0 & 1 & $\frac{q^{10}+q^{9}-3 q^{7}-13 q^{6}+17 q^{5}-q^{4}+5 q^{3}-4 q^{2}+1}{(q-1) q^{2}\left(q^{2}+1\right)\left(q^{2}+q+1\right)}$ & 1 & 1 & 1 & $\frac{2\left(3 q^{6}-q^{5}-8 q^{4}+4 q^{3}-q^{2}+7 q-2\right)}{(q-1) q\left(q^{2}+1\right)\left(q^{2}+q+1\right)}$ \\
\hline 1 & 0 & 2 & $\frac{2\left(q^{4}+3 q^{3}+q-6\right)}{\left(q^{2}+1\right)\left(q^{2}+q+1\right)}$ & 1 & 1 & 2 & $-\frac{q^{7}-3 q^{5}-4 q^{4}-7 q^{3}+9 q^{2}+q+5}{q^{3}\left(q^{2}+1\right)\left(q^{2}+q+1\right)}$ \\
\hline 1 & 0 & 3 & $\frac{q^{3}+q-4}{(q-1) q^{2}\left(q^{2}+q+1\right)}$ & 1 & 1 & 3 & $\frac{2\left(q^{3}-q^{2}-1\right)}{(q-1) q^{5}\left(q^{2}+q+1\right)}$ \\
\hline 2 & 0 & 0 & $\frac{q^{7}+q^{6}+q^{5}-3 q^{4}-8 q^{3}+q^{2}+1}{\left(q^{2}+1\right)\left(q^{2}+q+1\right)}$ & 2 & 1 & 0 & $\frac{2\left(2 q^{5}-4 q^{3}-q^{2}-q+1\right)}{q\left(q^{2}+1\right)\left(q^{2}+q+1\right)}$ \\
\hline 2 & 0 & 1 & $-\frac{2\left(q^{7}-3 q^{6}-2 q^{5}+5 q^{4}+q^{3}+7 q^{2}-8 q+1\right)}{(q-1) q\left(q^{2}+1\right)\left(q^{2}+q+1\right)}$ & 2 & 1 & 1 & $\frac{q^{11}-4 q^{9}+5 q^{8}+3 q^{7}-q^{5}-22 q^{4}+12 q^{3}-2 q^{2}+5 q-1}{(q-1) q^{4}\left(q^{2}+1\right)\left(q^{2}+q+1\right)}$ \\
\hline 2 & 0 & 2 & $-\frac{q^{7}+q^{6}+q^{5}-3 q^{4}-12 q^{3}+q^{2}-4 q+5}{q^{3}\left(q^{2}+1\right)\left(q^{2}+q+1\right)}$ & 2 & 1 & 2 & $-\frac{2\left(2 q^{7}-4 q^{5}-q^{4}-5 q^{3}+2 q^{2}+1\right)}{q^{6}\left(q^{2}+1\right)\left(q^{2}+q+1\right)}$ \\
\hline 2 & 0 & 3 & $\frac{2\left(q^{3}+q-1\right)}{(q-1) q^{5}\left(q^{2}+q+1\right)}$ & 2 & 1 & 3 & $\frac{4 q^{3}-q^{2}-1}{(q-1) q^{8}\left(q^{2}+q+1\right)}$ \\
\hline 3 & 0 & 0 & $\frac{2\left(q^{4}+q^{3}-1\right)}{\left(q^{2}+1\right)\left(q^{2}+q+1\right)}$ & 3 & 1 & 0 & $\frac{q^{4}+q^{3}+2 q^{2}+q-3}{q\left(q^{2}+1\right)\left(q^{2}+q+1\right)}$ \\
\hline 3 & 0 & 1 & $-\frac{q^{8}-4 q^{5}-2 q^{4}+8 q^{3}-2 q^{2}+4 q-1}{(q-1) q^{3}\left(q^{2}+1\right)\left(q^{2}+q+1\right)}$ & 3 & 1 & 1 & $-\frac{2\left(q^{6}-2 q^{5}-q^{4}+2 q^{3}-q^{2}+4 q-1\right)}{(q-1) q^{4}\left(q^{2}+1\right)\left(q^{2}+q+1\right)}$ \\
\hline 3 & 0 & 2 & $-\frac{2\left(q^{6}+q^{5}-2 q^{2}-1\right)}{q^{5}\left(q^{2}+1\right)\left(q^{2}+q+1\right)}$ & 3 & 1 & 2 & $-\frac{q^{4}+q^{3}+q^{2}-4}{q^{6}\left(q^{2}+q+1\right)}$ \\
\hline 3 & 0 & 3 & $\frac{q^{2}+1}{(q-1) q^{7}\left(q^{2}+q+1\right)}$ & 3 & 1 & 3 & $\frac{2}{(q-1) q^{8}\left(q^{2}+q+1\right)}$ \\
\hline
\end{tabular}

TABLE III. Coefficients of the function $\mathcal{H}$ for the FZ model with $L=2$.

\begin{tabular}{llllllll}
\hline \hline $\mathrm{i}$ & $\mathrm{j}$ & $\mathrm{k}$ & \multicolumn{1}{c}{$\phi_{i, j, k} / \phi_{0,0,0}$} & $\mathrm{i}$ & $\mathrm{j}$ & $\mathrm{k}$ & $\phi_{i, j, k} / \phi_{0,0,0}$ \\
\hline 0 & 0 & 0 & 1 & 0 & 1 & 0 & $-\frac{2}{q^{2}+1}$ \\
0 & 0 & 1 & $-\frac{2(q+2)}{q^{2}+1}$ & 0 & 1 & 1 & $\frac{q^{5}+5 q^{4}+9 q^{3}+4 q^{2}-1}{q^{3}\left(q^{2}+1\right)\left(q^{2}+q+1\right)}$ \\
0 & 0 & 2 & $\frac{(2 q+1)\left(q^{2}+4 q+1\right)}{q\left(q^{2}+1\right)\left(q^{2}+q+1\right)}$ & 0 & 1 & 2 & $-\frac{2\left(q^{4}+3 q^{3}+5 q^{2}+q-1\right)}{q^{3}\left(q^{2}+1\right)\left(q^{2}+q+1\right)}$ \\
0 & 0 & 3 & $-\frac{2}{q\left(q^{2}+1\right)}$ & 0 & 1 & 3 & $\frac{q^{2}+4 q+1}{q^{2}\left(q^{2}+1\right)\left(q^{2}+q+1\right)}$ \\
1 & 0 & 0 & $-\frac{2(q+1)}{q^{2}+1}$ & 1 & 1 & 0 & $\frac{(q+1)\left(q^{2}+4 q+1\right)}{q\left(q^{2}+1\right)\left(q^{2}+q+1\right)}$ \\
1 & 0 & 1 & $-\frac{(q+1)\left(q^{4}-q^{3}-8 q^{2}-9 q-1\right)}{q\left(q^{2}+1\right)\left(q^{2}+q+1\right)}$ & 1 & 1 & 1 & $-\frac{2(q+1)\left(3 q^{3}+5 q^{2}+2 q-1\right)}{q^{3}\left(q^{2}+1\right)\left(q^{2}+q+1\right)}$ \\
1 & 0 & 2 & $\frac{2(q+1)\left(q^{3}-2 q^{2}-5 q-3\right)}{q\left(q^{2}+1\right)\left(q^{2}+q+1\right)}$ & 1 & 1 & 2 & $\frac{(q+1)\left(q^{4}+9 q^{3}+8 q^{2}+q-1\right)}{q^{4}\left(q^{2}+1\right)\left(q^{2}+q+1\right)}$ \\
1 & 0 & 3 & $\frac{(q+1)\left(q^{2}+4 q+1\right)}{q^{2}\left(q^{2}+1\right)\left(q^{2}+q+1\right)}$ & 1 & 1 & 3 & $-\frac{2(q+1)}{q^{3}\left(q^{2}+1\right)}$ \\
\hline
\end{tabular}


TABLE III. (Continued).

\begin{tabular}{|c|c|c|c|c|c|c|c|}
\hline $\mathrm{i}$ & $\mathrm{j}$ & $\mathrm{k}$ & $\phi_{i, j, k} / \phi_{0,0,0}$ & $\mathrm{i}$ & $\mathrm{j}$ & $\mathrm{k}$ & $\phi_{i, j, k} / \phi_{0,0,0}$ \\
\hline 2 & 0 & 0 & $\frac{q^{2}+4 q+1}{\left(q^{2}+1\right)\left(q^{2}+q+1\right)}$ & 2 & 1 & 0 & $-\frac{2}{q\left(q^{2}+1\right)}$ \\
\hline 2 & 0 & 1 & $\frac{2\left(q^{4}-q^{3}-5 q^{2}-3 q-1\right)}{q\left(q^{2}+1\right)\left(q^{2}+q+1\right)}$ & 2 & 1 & 1 & $\frac{(q+2)\left(q^{2}+4 q+1\right)}{q^{2}\left(q^{2}+1\right)\left(q^{2}+q+1\right)}$ \\
\hline 2 & 0 & 2 & $-\frac{q^{5}-4 q^{3}-9 q^{2}-5 q-1}{q^{2}\left(q^{2}+1\right)\left(q^{2}+q+1\right)}$ & 2 & 1 & 2 & $-\frac{2(2 q+1)}{q^{3}\left(q^{2}+1\right)}$ \\
\hline 2 & 0 & 3 & $-\frac{2}{q^{2}\left(q^{2}+1\right)}$ & 2 & 1 & 3 & $\frac{1}{q^{4}}$ \\
\hline 3 & 0 & 0 & 0 & 3 & 1 & 0 & 0 \\
\hline 3 & 0 & 1 & 0 & 3 & 1 & 1 & 0 \\
\hline 3 & 0 & 2 & 0 & 3 & 1 & 2 & 0 \\
\hline 3 & 0 & 3 & 0 & 3 & 1 & 3 & 0 \\
\hline
\end{tabular}

TABLE IV. Coefficients of the function $\overline{\mathcal{H}}$ for the FZ model with $L=2$.

\begin{tabular}{|c|c|c|c|c|c|c|c|}
\hline $\mathrm{i}$ & $\mathrm{j}$ & $\mathrm{k}$ & $\bar{\phi}_{i, j, k} / \phi_{0,0,0}$ & $\mathrm{i}$ & j & $\mathrm{k}$ & $\bar{\phi}_{i, j, k} / \phi_{0,0,0}$ \\
\hline 0 & 0 & 0 & 1 & 0 & 1 & 0 & $-\frac{2 q}{q^{2}+1}$ \\
\hline 0 & 0 & 1 & $-\frac{2(q+1)}{q^{2}+1}$ & 0 & 1 & 1 & $\frac{(q+1)\left(q^{2}+4 q+1\right)}{\left(q^{2}+1\right)\left(q^{2}+q+1\right)}$ \\
\hline 0 & 0 & 2 & $\frac{q^{2}+4 q+1}{q^{4}+q^{3}+2 q^{2}+q+1}$ & 0 & 1 & 2 & $-\frac{2}{q^{2}+1}$ \\
\hline 0 & 0 & 3 & 0 & 0 & 1 & 3 & 0 \\
\hline 1 & 0 & 0 & $-\frac{4 q+2}{q^{2}+1}$ & 1 & 1 & 0 & $\frac{-q^{5}+4 q^{3}+9 q^{2}+5 q+1}{q^{4}+q^{3}+2 q^{2}+q+1}$ \\
\hline 1 & 0 & 1 & $\frac{(q+1)\left(q^{4}+9 q^{3}+8 q^{2}+q-1\right)}{q^{2}\left(q^{2}+1\right)\left(q^{2}+q+1\right)}$ & 1 & 1 & 1 & $\frac{2(q+1)\left(q^{3}-2 q^{2}-5 q-3\right)}{\left(q^{2}+1\right)\left(q^{2}+q+1\right)}$ \\
\hline 1 & 0 & 2 & $-\frac{2\left(q^{4}+3 q^{3}+5 q^{2}+q-1\right)}{q^{2}\left(q^{2}+1\right)\left(q^{2}+q+1\right)}$ & 1 & 1 & 2 & $\frac{2 q^{3}+9 q^{2}+6 q+1}{q^{5}+q^{4}+2 q^{3}+q^{2}+q}$ \\
\hline 1 & 0 & 3 & 0 & 1 & 1 & 3 & 0 \\
\hline 2 & 0 & 0 & $\frac{(q+2)\left(q^{2}+4 q+1\right)}{\left(q^{2}+1\right)\left(q^{2}+q+1\right)}$ & 2 & 1 & 0 & $\frac{2\left(q^{4}-q^{3}-5 q^{2}-3 q-1\right)}{\left(q^{2}+1\right)\left(q^{2}+q+1\right)}$ \\
\hline 2 & 0 & 1 & $-\frac{2(q+1)\left(3 q^{3}+5 q^{2}+2 q-1\right)}{q^{2}\left(q^{2}+1\right)\left(q^{2}+q+1\right)}$ & 2 & 1 & 1 & $\frac{-q^{5}+9 q^{3}+17 q^{2}+10 q+1}{q^{5}+q^{4}+2 q^{3}+q^{2}+q}$ \\
\hline 2 & 0 & 2 & $\frac{q^{5}+5 q^{4}+9 q^{3}+4 q^{2}-1}{q^{3}\left(q^{2}+1\right)\left(q^{2}+q+1\right)}$ & 2 & 1 & 2 & $-\frac{2(q+2)}{q^{3}+q}$ \\
\hline 2 & 0 & 3 & 0 & 2 & 1 & 3 & 0 \\
\hline 3 & 0 & 0 & $-\frac{2}{q^{2}+1}$ & 3 & 1 & 0 & $\frac{q^{2}+4 q+1}{q^{4}+q^{3}+2 q^{2}+q+1}$ \\
\hline 3 & 0 & 1 & $\frac{q^{3}+5 q^{2}+5 q+1}{q^{5}+q^{4}+2 q^{3}+q^{2}+q}$ & 3 & 1 & 1 & $-\frac{2(q+1)}{q^{3}+q}$ \\
\hline 3 & 0 & 2 & $-\frac{2}{q^{3}+q}$ & 3 & 1 & 2 & $\frac{1}{q^{2}}$ \\
\hline 3 & 0 & 3 & 0 & 3 & 1 & 3 & 0 \\
\hline
\end{tabular}

\section{REFERENCES}

${ }^{1}$ J. D. Bernal and R. H. Fowler, “A theory of water and ionic solution, with particular reference to hydrogen and hydroxyl ions,” J. Chem. Phys. 1, 515 (1933).

${ }^{2}$ L. Pauling, "The structure and entropy of ice and of other crystals with some randomness of atomic arrangement," J. Am. Chem. Soc. 57, 2680 (1935).

${ }^{3}$ J. C. Slater, "Theory of the transition in $\mathrm{KH}_{2} \mathrm{PO}_{4}$," J. Chem. Phys. 9, 16 (1941).

${ }^{4}$ F. Rys, "Über ein zweidimensionalles klassisches Konfigurationsmodell," Helv. Phys. Acta 36, 537 (1963).

${ }^{5}$ J. F. Nagle, "Lattice statistics of hydrogen bonded crystals. II. The Slater KDP model and the Rys F-model," J. Math. Phys. 7, 1492 (1966).

${ }^{6}$ R. J. Baxter, Exactly Solved Models in Statistical Mechanics (Dover Publications, Inc., Mineola, New York, 2007). 
${ }^{7}$ A. G. Izergin and V. E. Korepin, “The inverse scattering method approach to the quantum Shabat-Mikhailov model," Commun. Math. Phys. 79, 303-316 (1981).

${ }^{8}$ V. A. Fateev and A. B. Zamolodchikov, "Model factorized S matrix and an integrable Heisenberg chain with spin 1," Nucl. Phys. B 32, 298-303 (1980).

${ }^{9}$ V. V. Bazhanov and A. G. Shadrikov, "Trigonometric solutions of triangle equations. Simple Lie superalgebras," Theor. Math. Phys. 73, 1302 (1987).

${ }^{10}$ W. Galleas and M. J. Martins, " $R$-matrices and spectrum of vertex models based on superalgebras," Nucl. Phys. B 699(3), 455-486 (2004).

${ }^{11}$ W. Galleas and M. J. Martins, "New R-matrices from representations of braid-monoid algebras based on superalgebras," Nucl. Phys. B 732, 444-462 (2006).

${ }^{12}$ W.-L. Yang and Y. Zhen, "Bethe Ansatz for supersymmetric model constructed from $U_{q}\left[o s p(2 \mid 2)^{(2)}\right]$ R-matrix," Commun. Theor. Phys. 36(3), 381 (2001).

${ }^{13}$ V. V. Bazhanov, “Trigonometric solution of triangle equations and classical Lie algebras," Phys. Lett. B 159, 321-324 (1985).

${ }^{14} \mathrm{M}$. Jimbo, "Quantum R-matrix for the generalized Toda system," Commun. Math. Phys. 102, 537-547 (1986).

${ }^{15} \mathrm{~V}$. Korepin and P. Zinn-Justin, "Thermodynamic limit of the six-vertex model with domain wall boundary conditions," J. Phys. A: Math. Gen. 33(40), 7053-7066 (2000).

${ }^{16}$ H. A. Kramers and G. H. Wannier, "Statistics of the two-dimensional ferromagnet. Part I," Phys. Rev. 60(3), 252 (1941).

${ }^{17}$ H. A. Kramers and G. H. Wannier, "Statistics of the two-dimensional ferromagnet. Part II," Phys. Rev. 60(3), 263 (1941).

${ }^{18}$ V. O. Tarasov, "Algebraic Bethe Ansatz for the Izergin-Korepin R-matrix," Theor. Math. Phys. 76(2), 793-803 (1988).

${ }^{19}$ V. E. Korepin, "Calculation of norms of Bethe wave functions," Commun. Math. Phys. 86, 391-418 (1982).

${ }^{20}$ A. G. Izergin, "Partition function of the six-vertex model in a finite lattice," Sov. Phys. Dokl. 32, 878 (1987).

${ }^{21}$ P. M. Bleher and V. V. Fokin, "Exact solution of the six-vertex model with domain wall boundary conditions. Disordered phase," Commun. Math. Phys. 268(1), 223-284 (2006).

${ }^{22}$ P. M. Bleher and K. Liechty, "Exact solution of the six-vertex model with domain wall boundary conditions. Ferroelectric phase," Commun. Math. Phys. 286(2), 777-801 (2009).

${ }^{23}$ P. M. Bleher and K. Liechty, "Exact solution of the six-vertex model with domain wall boundary conditions: Antiferroelectric phase," Commun. Pure Appl. Math. 63(6), 779-829 (2010).

${ }^{24}$ H. Cohn, N. Elkies, and J. Propp, “Local statistics for random domino tilings of the Aztec diamond," Duke Math. J. 85(1), 117-166 (1996).

${ }^{25}$ F. Colomo and A. G. Pronko, "The arctic circle revisited," Contemp. Math. 458, 361-376 (2008).

${ }^{26}$ W. Galleas, "New differential equations in the six-vertex model," J. Stat. Mech. 2016(3), 33106-33118.

${ }^{27}$ W. Galleas, "On the elliptic $\mathfrak{g l}_{2}$ solid-on-solid model: Functional relations and determinants," J. Math. Phys. 60, 023503 (2019).

${ }^{28}$ W. Galleas, "Domain-wall boundaries through non-diagonal twists in the six-vertex model," J. Stat. Mech. 2019, 023101.

${ }^{29}$ W. Galleas, "Multiple integral representation for the trigonometric SOS model with domain wall boundaries," Nucl. Phys. B 858(1), 117-141 (2012).

${ }^{30}$ W. Galleas, "Refined functional relations for the elliptic SOS model," Nucl. Phys. B 867, 855-871 (2013).

${ }^{31}$ A. Caradoc, O. Foda, and N. Kitanine, "Higher spin vertex models with domain wall boundary conditions," J. Stat. Mech. 2006(03), P03012.

${ }^{32}$ A. Garbali, “The domain wall partition function for the Izergin-Korepin nineteen-vertex model at a root of unity," J. Stat. Mech. 2016(3), 033112.

${ }^{33}$ W. Galleas, "Functional relations for the six-vertex model with domain wall boundary conditions," J. Stat. Mech. 2010(06), P06008.

${ }^{34}$ W. Galleas, "A new representation for the partition function of the six-vertex model with domain wall boundaries," J. Stat. Mech. 2011(01), P01013.

${ }^{35} \mathrm{~W}$. Galleas, "Partition function of the elliptic solid-on-solid model as a single determinant," Phys. Rev. E 94(1), 010102 (2016).

${ }^{36}$ J.-S. Caux and J. Mossel, "Remarks on the notion of quantum integrability," J. Stat. Mech. 2011(02), P02023.

${ }^{37}$ E. K. Sklyanin, L. A. Takhtadzhyan, and L. D. Faddeev, "Quantum inverse problem method. I," Theor. Math. Phys. 40(2), 688-706 (1979).

${ }^{38}$ L. A. Takhtadzhyan and L. D. Faddeev, "The quantum method of the inverse problem and the Heisenberg XYZ model," Russ. Math. Surv. 34(5), 11 (1979).

${ }^{39}$ E. K. Sklyanin, “The quantum Toda chain,” Lect. Notes Phys. 226, 196-233 (1985).

${ }^{40}$ V. E. Korepin, N. M. Bogoliubov, and A. G. Izergin, Quantum Inverse Scattering Method and Correlation Functions (Cambridge University Press, 1993).

${ }^{41}$ G. Kuperberg, “Another proof of the alternating sign matrix conjecture," Int. Math. Res. Notices 1996(3), 139-150. 